\title{
EIGENPAIRS OF WILKINSON MATRICES *
}

\author{
CARLA FERREIRA $^{\dagger}$ AND BERESFORD PARLETT ${ }^{\ddagger}$
}

\begin{abstract}
In the 1950s J. H. Wilkinson introduced two families of symmetric tridiagonal integer matrices. Most of the eigenvalues are close to diagonal entries. We develop the structure of their eigenvectors in a natural way which reveals that these eigenvectors all look the same to the naked eye. The shape of the envelopes is like twin peaks (or a badly dented bell curve). We also analyze the eigenvectors of the remaining noninteger eigenvalues.
\end{abstract}

Key words. Wilkinson matrix, persymmetry, eigensystem

AMS subject classifications. $65 \mathrm{~F} 15$

1. Introduction. For each positive integer $m$, J. H. Wilkinson defined two real symmetric tridiagonal matrices $W_{2 m+1}^{ \pm}$as follows. All the next-to-diagonal entries are one. The diagonal entries are given by

$$
\operatorname{diag}\left(W_{2 m+1}^{ \pm}\right)=\left[\begin{array}{lllllllll}
m & m-1 & \ldots & 1 & 0 & \pm 1 & \pm 2 & \ldots & \pm m
\end{array}\right] .
$$

These matrices may be found in [15, pp. 308-311] but were circulating among the experts a decade earlier (see [16]). Each matrix illustrated subtle difficulties in the automatic computation of eigenvectors and eigenvalues as described in the next section. These matrices did not come from applications.

It seems that no one has studied the structure of the eigenvectors of these matrices and it was our observation that nearly all of them had the same shape that launched our investigations. More precisely, the visual support of the normalized eigenvectors of $W_{2 m+1}^{-}$is limited to 9 contiguous indices and the interior eigenvectors are displaced versions of each other, visually. The situation for $W_{2 m+1}^{+}$is more subtle; the visual support of a normalized eigenvector is confined to two sections of 9 contiguous indices. See Figures 1.1 and 1.2 together with Table 1.1. Most of the eigenvalues are integers to visual accuracy.

We should not have been surprised by these observations and Section 8 discusses what happens when the parameter $m$ reaches infinity. The shape revealed in Figure 1.1 is very special because it is orthogonal to all its displaced versions.

The paper is organized as follows. A little history gives the setting for the invention of each class of matrices and will be news to young readers. Next comes important notation and the concept of persymmetry. Our analysis begins with the triangular factorization $L D L^{t}$ of $V_{m}$, the leading principal $m \times m$ submatrix of both families. It is the rapid convergence, as $m \rightarrow \infty$, of the pivots in $D$ that drives the eigenvalues of all three matrices $V_{m}, W_{2 m+1}^{-}$and $W_{2 m+1}^{+}$towards integer values. Next we show the secular equations (rational expressions for the eigenvalues) because we find them so informative. The essential analysis is complete and we present, in Section 7 the structure of the eigenvectors for each family showing the similarities and differences. The final section, the big picture, lets $m$ reach infinity and reveals that $W_{\infty}^{-}$has the integers $\mathbb{Z}$ for its spectrum and all eigenvectors are displaced versions of each other. Both $V_{\infty}$ and $W_{\infty}^{+}$are also interesting. We had not considered $m=\infty$ when we began this paper. We thank two anonymous referees for their useful suggestions. In particular Section 8.1 comes from one of them.

\footnotetext{
* The research of Carla Ferreira author was partially financed by Portuguese Funds through FCT (Fundação para a Ciência e a Tecnologia) within the Project UID/MAT/00013/2013.

${ }^{\dagger}$ Centro de Matemática, Universidade do Minho, 4710-057 Braga, Portugal (caferrei@math.uminho.pt).

${ }^{\ddagger}$ Department of Mathematics and Computer Science Division of the EECS Department, University of California, Berkeley, California 94720-3840, U.S.A. (parlett@math.berkeley.edu).
} 
Carla Ferreira and Beresford Parlett

\begin{tabular}{|c|c|c|}
\hline$W_{31}^{-}$ & \multicolumn{2}{|c|}{$W_{31}^{+}$} \\
\hline 0 & & -1.12544152 \\
\hline \pm 1.00000000 & 0.25380582 & 0.94753437 \\
\hline \pm 2.00000000 & 1.78932135 & 2.13020922 \\
\hline \pm 3.00000000 & 2.96105888 & 3.04309929 \\
\hline \pm 4.00000000 & 3.99604800 & 4.00435382 \\
\hline \pm 5.00000000 & 4.99977432 & 5.00023627 \\
\hline \pm 6.00000000 & 5.99999184 & 6.00000836 \\
\hline \pm 7.00000000 & 6.99999980 & 7.00000021 \\
\hline \pm 8.00000000 & 8.00000000 & 8.00000001 \\
\hline \pm 9.00000021 & 9.00000021 & 9.00000021 \\
\hline \pm 10.00000816 & 10.00000816 & 10.00000816 \\
\hline \pm 11.00022568 & 11.00022568 & 11.00022568 \\
\hline \pm 12.00395200 & 12.00395200 & 12.00395200 \\
\hline \pm 13.03894112 & 13.03894112 & 13.03894112 \\
\hline \pm 14.21067865 & 14.21067865 & 14.21067865 \\
\hline \pm 15.74619418 & 15.74619418 & 15.74619418 \\
\hline
\end{tabular}
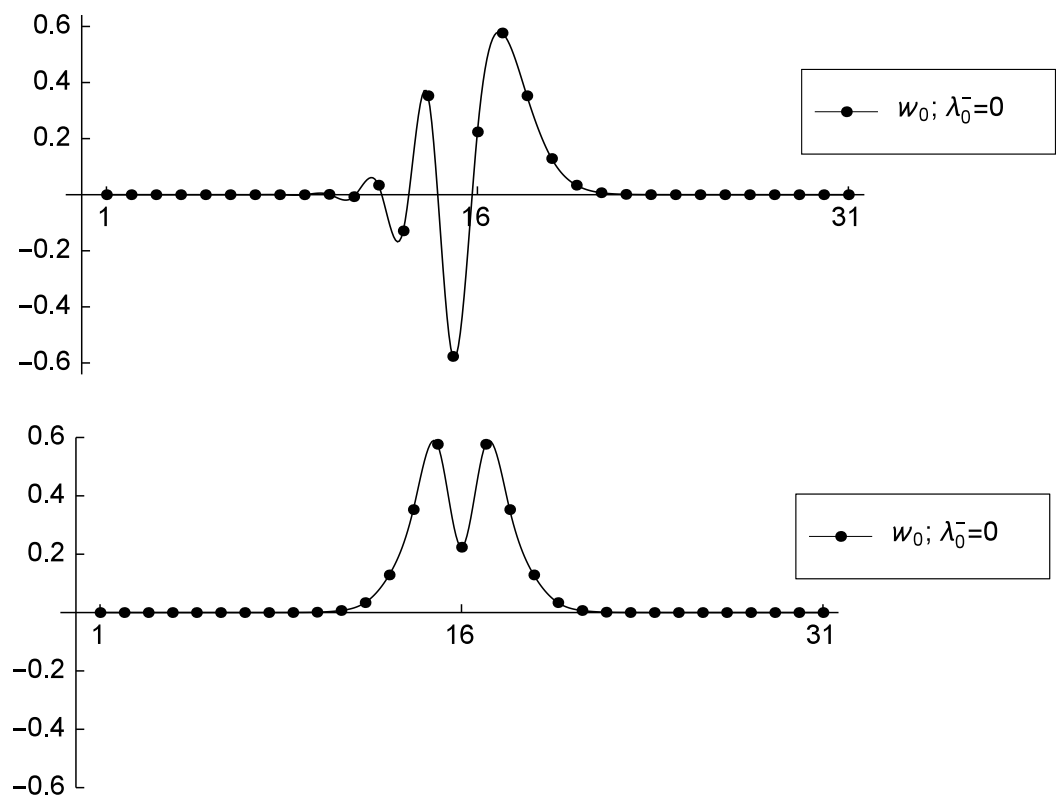

Figure 1.1. Eigenvector $\boldsymbol{w}_{0}$ of $W_{31}^{-}$for eigenvalue $\lambda_{0}^{-}=0$ (top) and its envelope (bottom).

The matrix $V_{m}$, mentioned above, is relevant as may be seen from the relation

$$
W_{2 m+1}^{-}=V_{2 m+1}-(m+1) I_{2 m+1} .
$$



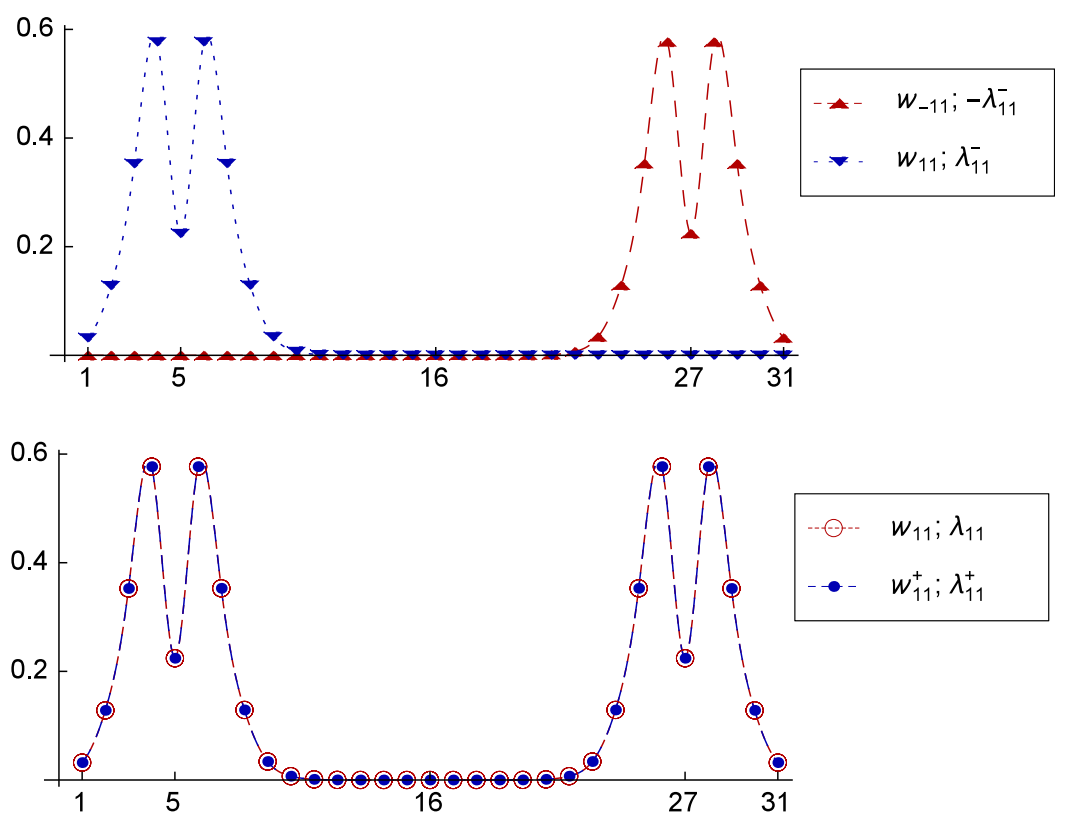

FigURE 1.2. $W_{31}^{-}$envelopes of eigenvectors $\boldsymbol{w}_{11}$ and $\boldsymbol{w}_{-11}$ for eigenvalues $\lambda_{11}^{-} \simeq 11$ and $-\lambda_{11}^{-} \simeq-11$, respectively (top). $W_{31}^{+}$envelopes of eigenvectors $\boldsymbol{w}_{11}$ and $\boldsymbol{w}_{11}^{+}$for eigenvalues close to $11, \lambda_{11}$ and $\lambda_{11}^{+}$, respectively (bottom).

Last but not least, we want to encourage the use of the attractive notation, introduced by G. W. (Pete) Stewart, for the reversal matrix,

$$
X_{m}=\left[\begin{array}{lllll} 
& & & & 1 \\
& & & 1 & \\
& & & & \\
1 & & & &
\end{array}\right] .
$$

We hope it becomes as ubiquitous as the use of $I_{m}$ for the identity matrix.

2. A little history. We look back to 1954 to a mostly forgotten piece of work by W. Givens. The 1950's were exciting times for the new enterprise of automatic eigenvalue computations. As early as 1950 C. Lanczos published his method of minimized iterations for reducing a full real symmetric matrix $A$ to tridiagonal form $T$. It was soon apparent that the tiny roundoff errors, inevitable in using computer arithmetic, caused significant changes from what was predicted by analysis of the algorithm in exact arithmetic and slowed its adoption. The amazing result of C. C. Paige that the eigenvalues are not degraded by these changes had to wait over 20 years till 1971 [8, 9, 10].

In another direction the method used by C. G. J. Jacobi, in 1846, for hand calculation of the eigenvalues of a $7 \times 7$ real symmetric matrix $A$ had been rediscovered [5] but was considered rather slow. The very sophisticated work of Z. Drmač to speed up the process to a competitive level did not appear until the $21^{\text {st }}$ century 3 .

So, what did W. Givens do? He proposed to reduce $A$ to $T$ by a well chosen sequence of plane rotations. The eigenvalues of $T$ he computed using bisection and Sturm sequences of polynomials 
from $T$. He gave the first formal backward error analysis in this field, showing that the computed eigenvalues belong to a matrix very close to $A$ in norm. In a backward analysis we do not discuss the errors in the computed eigenvalues. We must mention that Givens used fixed point arithmetic and it was already on its way out by the mid 1950's for matrix computations. However, at that time, fixed point analysis was considered much easier than error analysis of the new floating point arithmetic.

So far we have not mentioned eigenvectors. To compute an eigenvector $\boldsymbol{v}$ for a computed eigenvalue $\lambda$ of $T$, Givens used what we should call the pure mathematician's algorithm. It is not hard to see that $\boldsymbol{v}$ 's top entry $v_{1}$ can not vanish. So set it to 1 and use the top equation in $(T-\lambda I) \boldsymbol{v}=\mathbf{0}$ to determine $v_{2}$, use the second equation to determine $v_{3}$ and so on until the penultimate equation yields the last entry. There is no need to split the matrix into factors. Tridiagonal form is exploited to the hilt.

At this point Wilkinson's matrix $W_{21}^{-}$enters the picture. All of its eigenvalues are well separated and $\lambda_{\max } \simeq 10.75, \lambda_{\min } \simeq-10.75$. It's eigensystem is easy to compute. Using the method described above on his computer with precision roughly to 9 decimals, Wilkinson computed the corresponding eigenvectors $\boldsymbol{v}_{\text {max }}$ and $\boldsymbol{v}_{\text {min }}$. Although $\boldsymbol{v}_{\text {min }}$ was extremely accurate (even the tiny entries had most digits correct), the computed $\boldsymbol{v}_{\max }$ was pointing in the wrong direction. With this simple matrix, Wilkinson demonstrated unequivocally that this method (which unfortunately was called Givens method) was unreliable. This story is a little sad because if the last entry were set to 1 , instead of the first, and the equations in $\left(W_{21}^{-}-\lambda_{\max } I\right) \boldsymbol{v}_{\max }=\mathbf{0}$ had been used in reverse order to compute $\boldsymbol{v}_{\max }$ from bottom to top, then the output would have been excellent. The ultimately satisfactory algorithm did not appear until almost 2000 when numerical analysts accepted to factor $T-\lambda I$ both from top down and bottom up in order to decide on the right entry to set to 1 (see [11, 12] for details).

This story reveals how devastating a few roundoff errors can be in matrix computations, in contrast to some other parts of numerical analysis (pde boundary value problems) where roundoff error is dwarfed by discretization error.

Next we turn to $W_{21}^{+}$which was created to have eigenvalues in pairs with separation varying all the way from $10^{-15}$ up to 0.1 . A formidable test for any procedure. The natural "workhorse" to compute eigenvectors is to use some variant of inverse iteration; at step $i$ one solves $(T-\lambda I) \boldsymbol{v}^{i+1}=\boldsymbol{v}^{i}$ for the new vector $\boldsymbol{v}^{i+1}$. However, when eigenvalues that are formally distinct are computed equal to working accuracy, inverse iteration runs into trouble. Knowing how $W_{21}^{+}$is constructed gives rise to the idea that different parts of the matrix may be used to compute eigenvectors that are orthogonal and yet share the same eigenvalue. This idea is pursued in [13] and [4].

The challenge for real symmetric matrices is to produce eigenvectors orthogonal to working precision without getting trapped into invoking the Gram-Schmidt procedure too often.

\section{Preliminaries.}

3.1. Essential notation. $I_{n}=\left[\begin{array}{llll}e_{1} & \boldsymbol{e}_{2} & \ldots & \boldsymbol{e}_{n}\end{array}\right]$. We introduced the symbol $\quad I$ for the reversal matrix in Section $1 . x_{m}=\left[\begin{array}{llll}\boldsymbol{e}_{n} & \boldsymbol{e}_{n-1} & \ldots & \boldsymbol{e}_{1}\end{array}\right]$. The symmetries apparent on the diagonal of both $W_{2 m+1}^{-}$and $W_{2 m+1}^{+}$encourage us to look for eigenvectors having a matching structure. Recall the matrix $V_{m}$ from the Introduction and note that

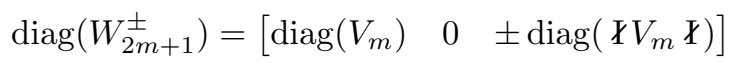

where $\chi$ takes its size from the context. Unfortunately the matrix $-\chi V_{m} X$ has all its next-todiagonal entries negative. This blemish may be removed by a notational trick. For any signature matrix $S(S=\operatorname{diag}( \pm 1))$ the matrix $S T S$ is similar to tridiagonal $T$, since $S=S^{-1}$. In this paper 
we fix $S$ to have alternating signs

$$
S_{m}=\operatorname{diag}\left(1,-1,1,-1, \ldots,(-1)^{m-1}\right)
$$

and to satisfy $S \boldsymbol{e}_{1}=\boldsymbol{e}_{1}$ for all $\mathrm{m}$. It is easily verified that $S T S$ reverses the signs of all next-todiagonal entries and leaves the diagonal invariant. Thus the trailing $m \times m$ principal submatrix of $W_{2 m+1}^{-}$may be written as $-S \Varangle V_{m} \backslash S$. This looks clumsy at first but permits simple analysis in all that follows. As with $X$ the context dictates the size of $S$. Note that $S^{2}=X^{2}=I$ and $S X= \pm X S$ ( $S \Varangle=\Varangle S$, for $m$ odd, and $S \Varangle=-\Varangle S$, for $m$ even). We use these relations without explicit mention throughout this work. In particular, with dimension $k, \chi S \boldsymbol{e}_{1}=\chi \boldsymbol{e}_{1}=\boldsymbol{e}_{k}$.

Lower case Roman letters denote column vectors and $\boldsymbol{v}^{t}$ is the transpose of $\boldsymbol{v}$. We use braces $\{\cdot\}$ for sequences or sets.

Another special notation in this paper is $\delta_{m}$ to denote the last pivot in the triangular factorization of $V_{m}$. Write $V_{m}=L D L^{t}, L$ unit lower bidiagonal, $D=\operatorname{diag}\left(d_{1}, d_{2}, \ldots, d_{m}\right)$ and $\delta_{m}=d_{m}$ here. The rapid convergence of $\delta_{m}$ to a limit $\phi>0$ explains our surprising observation that most of the eigenvectors of $V_{m}$ look the same to the naked eye and seem independent of $m$ !

Later we show that $V_{m}$ is positive definite. The eigenpairs of $V_{m}$ are denoted by

$$
V_{m} \boldsymbol{z}_{k}=\boldsymbol{z}_{k} \lambda_{k}, \quad k=1,2, \ldots, m, \quad 0=\lambda_{0}<\lambda_{1}<\lambda_{2}<\cdots<\lambda_{m} .
$$

Here the eigenvectors $\left\{\boldsymbol{z}_{i}\right\}$ are chosen orthonormal in contrast to our choice later of the eigenvectors of $W_{2 m+1}^{ \pm}$.

We will see that zero is an eigenvalue of $W_{2 m+1}^{-}$and that the other $2 m$ eigenvalues occur in \pm pairs. We denote these eigenvalues by $\lambda_{0}^{-}=0$ and $\pm \lambda_{k}^{-}, k=1, \ldots, m$, and represent the eigenvector equations as

$$
\begin{aligned}
& W_{2 m+1}^{-} \boldsymbol{w}_{k}=\boldsymbol{w}_{k} \lambda_{k}^{-}, \quad k=0,1,2, \ldots, m, \quad 0<\lambda_{1}^{-}<\lambda_{2}^{-}<\cdots<\lambda_{m}^{-} ; \\
& W_{2 m+1}^{-} \boldsymbol{w}_{-k}=\boldsymbol{w}_{-k}\left(-\lambda_{k}^{-}\right), \quad k=1,2, \ldots, m .
\end{aligned}
$$

We will show that each eigenvalue $\lambda_{k}$ of $V_{m}$ is an eigenvalue of $W_{2 m+1}^{+}$and the other $m+1$ eigenvalues will be denote by $\lambda_{k}^{+}, k=0, \ldots, m$. The eigenvector equations are

$$
\begin{aligned}
& W_{2 m+1}^{+} \boldsymbol{w}_{k}^{+}=\boldsymbol{w}_{k}^{+} \lambda_{k}^{+}, \quad k=0,1,2, \ldots, m, \quad \lambda_{0}^{+}<0<\lambda_{1}^{+}<\lambda_{2}^{+}<\cdots<\lambda_{m}^{+} ; \\
& W_{2 m+1}^{+} \boldsymbol{w}_{k}=\boldsymbol{w}_{k} \lambda_{k}, \quad k=1,2, \ldots, m .
\end{aligned}
$$

We acknowledge that the notation $\boldsymbol{w}_{k}$ will be used both for $W_{2 m+1}^{-}$and $W_{2 m+1}^{+}$, but the different expressions will appear in different sections of the paper and so this will not be a source of ambiguity.

For future reference we write down, in block form, the useful observation

$$
W_{2 m+1}^{ \pm}=\left[\begin{array}{ccc}
V_{m} & \boldsymbol{e}_{m} & O \\
\boldsymbol{e}_{m}^{t} & 0 & \boldsymbol{e}_{1}^{t} \\
O & \boldsymbol{e}_{1} & \pm \chi \widetilde{V}_{m} \chi
\end{array}\right], \quad \widetilde{V}_{m}=\left\{\begin{array}{l}
V_{m}, \text { for } W_{2 m+1}^{+} \\
S V_{m} S, \text { for } W_{2 m+1}^{-}
\end{array}\right.
$$

Notice that $S \Varangle V_{m} \backslash S=( \pm \Varangle S) V_{m}( \pm S X)=\Varangle S V_{m} S X$. In this context we can treat $S$ and 1 as though they always commute, which they do not.

The key to our analysis is that when $W_{2 m+1}^{-}$is shifted by a suitable integer $\pm k, k=1, \ldots, m$, the diagonal blocks in 3.1 break up into the blocks $V_{m-k}$ and $-1 S V_{m+k} S X$. The vector $V_{m-k}^{-1} \boldsymbol{e}_{m-k}$ is ubiquous in the rest of this paper. 
We also need to consider related vectors, say $\boldsymbol{w}^{(p)}$ and $\boldsymbol{w}$, for integer $p$, where $w^{(p)}(i)=w(i-p)$ for all entries $i$. We will say that $\boldsymbol{w}^{(p)}$ is a displaced version of $\boldsymbol{w}$ and we will usually not worry about end effects since most of our vectors decay rapidly near the extremes, top and bottom.

To keep things simple we will often show the envelope of an eigenvector, that is, the vector of the absolute values in place of the true eigenvector.

\subsection{Persymmetry.}

Definition 3.1. A real vector $\boldsymbol{v}$ is persymmetric (respectively, skew-persymmetric) if $\boldsymbol{x} \boldsymbol{v}=\boldsymbol{v}$ (respectively, $X \boldsymbol{v}=-\boldsymbol{v}$ ).

Definition 3.2. A real square matrix $M$ is persymmetric (respectively, skew-persymmetric) if $Y M Y=M^{t}$ (respectively, $Y M Y=-M^{t}$ ).

LEMma 3.3. If a real symmetric matrix is also persymmetric then each eigenvector $\boldsymbol{v}$ for a simple eigenvalue $\lambda$ is either persymmetric or skew-persymmetric.

Proof. Let real symmetric matrix $M$ satisfy $M \boldsymbol{v}=\boldsymbol{v} \lambda$, with $\lambda$ simple, and also be persymmetric. Observe that

$$
M(\Varangle \boldsymbol{v})=\Varangle(\Varangle M \Varangle \boldsymbol{v})=\Varangle M^{t} \boldsymbol{v}=\Varangle M \boldsymbol{v}=(\Varangle \boldsymbol{v}) \lambda .
$$

Since $\lambda$ is simple, $X \boldsymbol{v}$ is a multiple of $\boldsymbol{v}$, and, since $X$ is orthogonal, the only freedom is $\chi \boldsymbol{v}= \pm \boldsymbol{v}$. Both cases arise.

Note that $W_{2 m+1}^{-}$is not skew-persymmetric which is why we brought in our special signature matrix $S$. Fortunately,

$$
X S W_{2 m+1}^{-} S X=-W_{2 m+1}^{-},
$$

which is the appropriate replacement.

LEMmA 3.4. If a real symmetric matrix $M$ also satisfies $S Y M Y S=-M$, then its nonzero eigenvalues occur in \pm pairs.

Proof. Let $M \boldsymbol{v}=\boldsymbol{v} \lambda$, with $\lambda>0$ and simple. Then

$$
M(\chi S \boldsymbol{v})=\Varangle S(S \Varangle M \Varangle S \boldsymbol{v})=\Varangle S(-M) \boldsymbol{v}=(\Varangle S \boldsymbol{v})(-\lambda),
$$

which exhibits the negative eigenvalues $-\lambda$.

4. $V_{m}$ and its triangular factorizaton. From the Introduction (Section 1) recall that all the next-to-diagonal entries of $V_{m}$ are 1 and

$$
\operatorname{diag}\left(V_{m}\right)=\left[\begin{array}{llllll}
m & m-1 & m-2 & \cdots & 2 & 1
\end{array}\right] .
$$

$V_{m}$ is symmetric positive definite (see Section 5.1) and so permits triangular factorization in the form $V_{m}=L D L^{t}$, where $L$ is unit lower bidiagonal and $D=\operatorname{diag}\left(d_{1}, d_{2}, \ldots, d_{m}\right)$ holds the pivots $\left\{d_{j}\right\}$. The recurrence for the pivots and multipliers $\left\{l_{j}\right\}$ is

$$
d_{1}=m ; \quad d_{j+1}=m-j-\frac{1}{d_{j}}, \quad l_{j}=\frac{1}{d_{j}}, \quad j=1,2, \ldots, m-1 .
$$

Since $D$ is congruent to $V_{m}$ all the pivots are positive. We choose to present our analysis in a sequence of formal lemmas. Each lemma plays a significant role.

LEMMA 4.1. Given $V_{m}=L D L^{t}$,

$$
0 \leq m-j<d_{j} \leq m-j+1, \quad j=1,2, \ldots, m .
$$


Proof. (by induction). The basis is $d_{1}=m>m-1$. The induction assumption is that Lemma 4.1 holds for some $j<m$. In that case $d_{j}>m-j \geq 1$ and so

$$
d_{j+1}=m-j-\frac{1}{d_{j}}>m-j-1=m-(j+1)
$$

and, again by 4.1,,$d_{j+1}<m-j$. Thus Lemma 4.1 holds for the next value of $j$, namely $j+1$. By the principle of finite induction Lemma 4.1 holds for all $j=1,2, \ldots, m$. Equality in the upper bound holds only for $j=1$.

Lemma 4.1 is, in fact, the most direct proof that $V_{m}$ is positive definite. It says that the pivots $d_{j}, j=1, \ldots, m$, in the recurrence (4.1) are all positive which means that $V_{m}$ admits the triangular factorization $V_{m}=L D L^{t}$ (no need to assume it exists). Since $V_{m}$ is congruent to $D$, it follows that all the eigenvalues of $V_{m}$ are positive.

We need a special notation for the last pivot $d_{m}$ of each $V_{m}$. We choose

$$
\delta_{m}:=d_{m}\left(V_{m}\right) .
$$

In words, $\delta_{m}$ is the last element on $D$ 's diagonal for $V_{m}=L D L^{t}$. Note that $\delta_{m-1}=d_{m-1}\left(V_{m-1}\right)$ is different from $d_{m-1}=d_{m-1}\left(V_{m}\right)$.

By Lemma 4.1. $0<\delta_{m} \leq 1$.

Lemma 4.2. $\left[V_{m}^{-1}\right]_{m, m}=\delta_{m}^{-1}$.

Proof. Since $L$ is unit lower bidiagonal,

$$
\left[V_{m}^{-1}\right]_{m, m}=\boldsymbol{e}_{m}^{t}\left(L^{-t} D^{-1} L^{-1}\right) \boldsymbol{e}_{m}=\boldsymbol{e}_{m}^{t} D^{-1} \boldsymbol{e}_{m}=\left(d_{m}\left(V_{m}\right)\right)^{-1}=\delta_{m}^{-1} \cdot \square
$$

Lemma 4.3. The sequence $\left\{\delta_{m}\right\}$ is monotone decreasing.

Proof. For the duration of the next two proofs let $\bar{d}_{j}:=d_{j}\left(V_{m-1}\right), j=1, \ldots, m-1$. Compare $d_{j+1}$ with $\bar{d}_{j}$. For $j<m$, by 4.1

$$
\begin{aligned}
d_{j+1} & =(m-j)-\frac{1}{d_{j}}, \\
\bar{d}_{j} & =(m-1)-(j-1)-\frac{1}{\bar{d}_{j-1}}=(m-j)-\frac{1}{\bar{d}_{j-1}}, \quad j>1, \\
\bar{d}_{j}-d_{j+1} & =\frac{1}{d_{j}}-\frac{1}{\bar{d}_{j-1}}=\frac{\bar{d}_{j-1}-d_{j}}{d_{j} \bar{d}_{j-1}}, \quad j=1, \ldots, m-1 .
\end{aligned}
$$

Note that $d_{2}=m-1-\frac{1}{m}<m-1=\bar{d}_{1}$. By Lemma $4.1, d_{j}>0$ and $\bar{d}_{j-1}>0$, for all $1<j<m$, and thus we have $d_{j} \bar{d}_{j-1}>0$. So, (4.4) shows that $\bar{d}_{j}-d_{j+1}>0$ for all $j<m$, using the principle of finite induction. In particular,

$$
\delta_{m}=d_{m}\left(V_{m}\right)=d_{m}<\bar{d}_{m-1}=d_{m-1}\left(V_{m-1}\right)=\delta_{m-1},
$$

as claimed.

LEMmA 4.4. $\frac{2}{m !(m-1) !}<\delta_{m-1}-\delta_{m}<\frac{1}{m !(m-3) !}, \quad m>3$. 
Proof. For $m>3$, apply the recurrence 4.4 in the proof of Lemma 4.3 consecutively, in reverse, for $j=m-1, m-2, \ldots, 2,1$ to find

$$
\begin{aligned}
\delta_{m-1}-\delta_{m} & =\bar{d}_{m-1}-d_{m} \\
& =\frac{\bar{d}_{m-2}-d_{m-1}}{d_{m-1} \bar{d}_{m-2}}=\frac{\bar{d}_{m-3}-d_{m-2}}{d_{m-1} \bar{d}_{m-2} d_{m-2} \bar{d}_{m-3}}=\cdots \\
& =\frac{\bar{d}_{1}-d_{2}}{d_{m-1} \bar{d}_{m-2} d_{m-2} \bar{d}_{m-3} \cdots d_{3} \bar{d}_{2} d_{2} \bar{d}_{1}} \\
& =\frac{\bar{d}_{1}-d_{2}}{d_{m-1}\left(\prod_{i=2}^{m-2} \bar{d}_{m-i} d_{m-i}\right) \bar{d}_{1}}, \quad m>3 .
\end{aligned}
$$

Lemma 4.1 shows that for $m>3$,

$$
\frac{1}{2}(m-2) !(m-1) !=\prod_{i=2}^{m-2} i(i+1)>\prod_{i=2}^{m-2} \bar{d}_{m-i} d_{m-i}>\prod_{i=2}^{m-2}(i-1) i=(m-3) !(m-2) !
$$

Since $\bar{d}_{1}=m-1, d_{2}=m-1-1 / m$ and $d_{m-1}>1$,

$$
\frac{2}{m !(m-1) !}<\delta_{m-1}-\delta_{m}<\frac{1}{m(m-1)(m-2) !(m-3) !}=\frac{1}{m !(m-3) !},
$$

as claimed.

For $m=3$, we have $2 /[m !(m-1) !]=1 /[m !(m-3) !]=1 / 6$, but the strict upper bound still holds, $\delta_{2}-\delta_{3}=1 / 2-2 / 5=1 / 10<1 / 6$.

Since the sequence $\left\{\delta_{m}\right\}$ is bounded below by 0 and monotone decreasing, it converges to some limit

$$
\phi:=\lim _{m \rightarrow+\infty} \delta_{m} \geq 0
$$

What is important for our observation concerning the envelopes of the interior eigenvectors of $W_{2 m+1}^{-}$ is the rapid convergence of $\delta_{m}$ to $\phi$.

Lemma 4.5. For $m>2,0<\delta_{m}-\phi<\frac{1}{m !(m-1) !}$.

Proof. Write down Lemma 4.4 with increasing values. For a given whole number $m>2$,

$$
\begin{aligned}
\delta_{m}-\delta_{m+1} & <\frac{1}{(m+1) !(m-2) !}, \\
\delta_{m+1}-\delta_{m+2} & <\frac{1}{(m+2) !(m-1) !}=\frac{1}{(m+2)(m-1)(m+1) !(m-2) !}<\frac{m^{-2}}{(m+1) !(m-2) !} .
\end{aligned}
$$

Crudely, bounding below both $(m+j+1) \cdots(m+2)$ and $(m+j-2) \cdots(m-1)$ by $m^{j}$, for $j \geq 4$,

$$
\delta_{m+j}-\delta_{m+j+1}<\frac{1}{(m+j+1) !(m+j-2) !}<\frac{m^{-2 j}}{(m+1) !(m-2) !} .
$$


Sum these inequalities to obtain

$$
\begin{aligned}
\delta_{m}-\delta_{m+j+1} & =\delta_{m}-\delta_{m+1}+\delta_{m+1}-\delta_{m+2}+\ldots+\delta_{m+j}-\delta_{m+j+1} \\
& <\frac{1}{(m+1) !(m-2) !} \sum_{i=0}^{j} m^{-2 i}=\frac{1-m^{-2(j+1)}}{1-m^{-2}} \cdot \frac{1}{(m+1) !(m-2) !} \\
& <\frac{m^{2}}{m^{2}-1} \cdot \frac{1}{(m+1) !(m-2) !}=\frac{m^{2}}{(m+1)^{2}} \cdot \frac{1}{m !(m-1) !} \\
& <\frac{1}{m !(m-1) !}, \quad j \geq 0 .
\end{aligned}
$$

Now let $j \rightarrow+\infty$, to find

$$
\delta_{m}-\phi<\frac{1}{m !(m-1) !}
$$

as claimed.

Numerical evaluation of $\delta_{m}$ for increasing values of $m$ shows that

$$
\phi=0.3882107655 \ldots
$$

Using recurrence (4.1) in reverse gives a continued fraction representation for $\delta_{m}$. In a notation related to the Pringsheim's notation,

$$
\delta_{m}=1-\frac{1}{2-} \frac{1}{3-} \frac{1}{4-} \cdots \frac{1}{(m-1)-} \frac{1}{m} .
$$

We thank David H. Bailey and Karl Dilcher who pointed out to us (see [1]) that there is a closed form for $\phi$,

$$
\phi=\lim _{m \rightarrow+\infty} \delta_{m}=\frac{J_{0}(2)}{J_{1}(2)},
$$

where $J_{n}(z)$ is the Bessel function of the first kind (bounded at $x=0$ ) defined as the solution of Bessel's differential equation

$$
x^{2} \frac{d^{2} y}{d x^{2}}+x \frac{d y}{d x}+\left(x^{2}-n^{2}\right) y=0, \quad x \geq 0 .
$$

For full details see 14 .

Table 4.1 compares our bound, Lemma 4.5, to the real difference.

It is Lemma 4.5 that ensures that most eigenvalues of $V_{m}$ are integers. Section 5.2 gives the details (see Theorem 5.1).

4.1. Last column of $\boldsymbol{V}_{\boldsymbol{m}}^{-1}$ and its norm $\left\|\boldsymbol{V}_{\boldsymbol{m}}^{-1} \boldsymbol{e}_{\boldsymbol{m}}\right\|$. Let $\boldsymbol{x}=V_{m}^{-1} \boldsymbol{e}_{m}$ and use $V_{m}=L D L^{t}$ to find

$$
L^{t} \boldsymbol{x}=D^{-1} L^{-1} \boldsymbol{e}_{m}=D^{-1} \boldsymbol{e}_{m}=\boldsymbol{e}_{m} \delta_{m}^{-1}, \quad x(m)=\delta_{m}^{-1} \geq 1 .
$$

Back solve this bidiagonal system to find

$$
\begin{array}{r}
x(j)+l_{j} x(j+1)=0, \quad 1 \leq j<m, \\
x(m-j)=x(m) \prod_{i=1}^{j}\left(-l_{m-i}\right) .
\end{array}
$$




\begin{tabular}{ccc}
\hline$m$ & $\delta_{m}-\phi$ & $1 /[m !(m-1) !]$ \\
\hline 2 & $0.1118 \times 10^{0}$ & $0.500 \times 10^{0}$ \\
3 & $0.1179 \times 10^{-1}$ & $0.833 \times 10^{-1}$ \\
4 & $0.6781 \times 10^{-3}$ & $6.944 \times 10^{-3}$ \\
5 & $0.2453 \times 10^{-4}$ & $3.472 \times 10^{-4}$ \\
6 & $0.6165 \times 10^{-6}$ & $1.157 \times 10^{-5}$ \\
7 & $0.1145 \times 10^{-7}$ & $2.756 \times 10^{-7}$ \\
8 & $0.1638 \times 10^{-9}$ & $4.291 \times 10^{-9}$ \\
9 & $0.1859 \times 10^{-11}$ & $6.835 \times 10^{-11}$ \\
10 & $0.1721 \times 10^{-13}$ & $7.594 \times 10^{-13}$ \\
\hline \multicolumn{3}{c}{ TABLE 4.1 } \\
\multicolumn{3}{c}{ Convergence rate of $\delta_{m}$}
\end{tabular}

From (4.1) $l_{j}=1 / d_{j}>0$ and so the entries in $\boldsymbol{x}$ alternate in sign with $x(m) \geq 1>0$ always. LEMma 4.6. For $1 \leq j<m, \frac{1}{(j+1) !}<|x(m-j)|<\frac{1}{j ! \phi}<\frac{3}{j !}$.

Proof. By the remarks above

$$
|x(m-j)|=x(m) \prod_{i=1}^{j}\left|l_{m-i}\right|=\frac{1}{\delta_{m} \prod_{i=1}^{j} d_{m-i}} .
$$

By Lemma 4.1, $i<d_{m-i} \leq i+1, i=1, \ldots, m-1$. So

$$
j !<\prod_{i=1}^{j} d_{m-i}<(j+1) !
$$

Inequality is strict since $d_{m-i}=i+1$ only for $i=m-1$. Since $\frac{1}{3}<\phi<\delta_{m} \leq 1$,

$$
\frac{1}{(j+1) !}<|x(m-j)|<\frac{1}{j ! \phi}<\frac{3}{j !},
$$

as claimed. Notice that for $j=0$, we have $1 \leq x(m)<3$. $\square$

Thus, for instance, $|x(m-5)|<3 /(5 !)=0.025$ and

$$
\delta_{m}^{-2}+\frac{1}{(2 !)^{2}}+\frac{1}{(3 !)^{2}}+\text { smaller terms }<\|\boldsymbol{x}\|^{2}<\phi^{-2}\left(1+1+\frac{1}{(2 !)^{2}}+\text { smaller terms }\right) .
$$

But we can obtain more information about $\boldsymbol{x}=V_{m}^{-1} \boldsymbol{e}_{m}$. We use $\delta_{m} \longrightarrow \phi$ rapidly as $m$ increases. We already know $x(m)=d_{m}^{-1}=\delta_{m}^{-1} \simeq \phi^{-1}$ and, from 4.1], $d_{j}=\left(m-j-d_{j+1}\right)^{-1}$, $j=1,2, \ldots, m-1$. Thus,

$$
\begin{aligned}
& d_{m-1}=\left(1-d_{m}\right)^{-1} \simeq 1 /(1-\phi) \\
& d_{m-2}=\left(2-d_{m-1}\right)^{-1} \simeq(1-\phi) /(1-2 \phi) \\
& d_{m-3}=\left(3-d_{m-2}\right)^{-1} \simeq(1-2 \phi) /(2-5 \phi) \\
& d_{m-4}=\left(4-d_{m-3}\right)^{-1} \simeq(2-5 \phi) /(7-18 \phi)
\end{aligned}
$$


and

$$
\begin{aligned}
d_{m-1} d_{m-2} & \simeq 1 /(1-2 \phi) \\
d_{m-1} d_{m-2} d_{m-3} & \simeq 1 /(2-5 \phi) \\
d_{m-1} d_{m-2} d_{m-3} d_{m-4} & \simeq 1 /(7-18 \phi) .
\end{aligned}
$$

From (4.7),

$$
x(m-j)=x(m) \prod_{i=1}^{j}\left(-d_{m-i}^{-1}\right), \quad j=1,2,3,4 .
$$

Finally, componentwise, using 4.9 ,

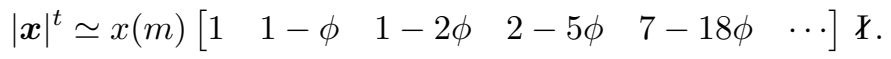

Thus, for $m \geq 5$,

$$
\begin{aligned}
\|\boldsymbol{x}\|^{2} & \simeq \phi^{-2}\left[1+(1-\phi)^{2}+(1-2 \phi)^{2}+(2-5 \phi)^{2}+(7-18 \phi)^{2}+\cdots\right] \\
& \simeq 9.47467+\cdots .
\end{aligned}
$$

From numerical calculations it turns out that $\|\boldsymbol{x}\|^{2}=8.78,9.42857 \ldots, 9.47291 \ldots, 9.47460 \ldots$, for $m=3,4,5,6$, respectively.

Figure 4.1 shows, in reverse order, the entries $x_{m-j}, j=0,1, \ldots, m-1$, of the last column $\boldsymbol{x}$ of $V_{m}^{-1}$ for $m=10,20,30,40$. They are indistinguishable. See also Table 4.2 .

For later reference please note that the vector $\boldsymbol{x}$ is well defined when $m=\infty$ and the entries are functions of $\phi$ as shown above in (4.10).

We note here that there are some other studies on the decay of off-diagonal elements when inverting tridiagonal or bidiagonal matrices, e.g., the one of R. Nabben [7] and the references therein.

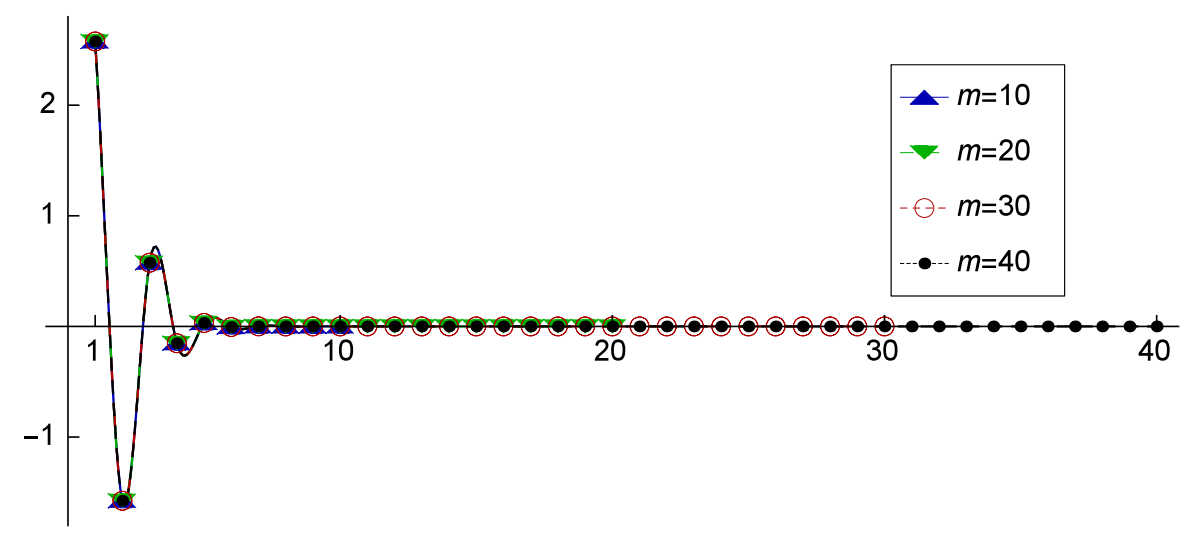

FIGURE 4.1. Last column (reversed) of $V_{m}^{-1}, m=10,20,30,40$. They are indistinguishable.

5. Eigenpairs of $\boldsymbol{V}_{\boldsymbol{m}}$. We describe the eigenpairs of $W_{2 m+1}^{ \pm}$using the eigenpairs of $V_{m}$, so this section is essential supporting material for our results. On the other hand $W_{2 m+1}^{-}=V_{2 m+1}-(m+1) I_{2 m+1}$ and so $W_{2 m+1}^{-}$has the same eigenvectors as $V_{2 m+1}$. The canonical eigenvector of $W_{2 m+1}^{-}$is the eigenvector for the eigenvalue $\mu=0$, which is the eigenvector for $\lambda_{m+1}=m+1$ of $V_{2 m+1}$, meaning that all the other eigenvectors look like displaced versions of this eigenvector. That leaves only $W_{2 m+1}^{+}$to be analyzed in Section 7.2 . 


\begin{tabular}{ccc}
\hline$m=5$ & $m=10$ & $m=20$ \\
\hline 2.5757576 & 2.5759203 & 2.5759203 \\
-1.5757576 & -1.5759203 & -1.5759203 \\
0.57575758 & 0.57592032 & 0.57592032 \\
-0.15151515 & -0.15184064 & -0.15184064 \\
$0.30303030 \times 10^{-1}$ & $0.31442250 \times 10^{-1}$ & $0.31442250 \times 10^{-1}$ \\
- & $-0.53706051 \times 10^{-2}$ & $-0.53706051 \times 10^{-2}$ \\
- & $0.78138131 \times 10^{-3}$ & $0.78138133 \times 10^{-3}$ \\
- & $-0.99064012 \times 10^{-4}$ & $-0.99064161 \times 10^{-4}$ \\
- & $0.11130788 \times 10^{-4}$ & $0.11131961 \times 10^{-4}$ \\
- & $-0.11130788 \times 10^{-5}$ & $-0.11234881 \times 10^{-5}$ \\
- & - & $0.10292003 \times 10^{-6}$ \\
- & - & $-0.86323124 \times 10^{-8}$ \\
- & - & $0.66771039 \times 10^{-9}$ \\
- & - & $-0.47922762 \times 10^{-10}$ \\
- & - & $0.32082681 \times 10^{-11}$ \\
- & - & $-0.20125911 \times 10^{-12}$ \\
- & - & $0.11877701 \times 10^{-13}$ \\
- & - & $-0.66181252 \times 10^{-15}$ \\
- & - & $0.34924143 \times 10^{-16}$ \\
- & TABLE 4.2 & $-0.17462071 \times 10^{-17}$ \\
\hline \multirow{2}{*}{ Entries of the last column (reversed) of $V_{m}^{-1}$ for $m=5,10,20}$.
\end{tabular}

5.1. Eigenvalues of $\boldsymbol{V}_{\boldsymbol{m}}$. An indirect proof that $V_{m}$ is positive definite comes from Gershgorin's Circle Theorem which implies that the closed interval $[0, m+1]$ contains $V_{m}$ 's spectrum, together with a more esoteric result which says that if a square matrix is irreducible and an eigenvalue $\lambda$ lies on the boundary of any Gershgorin disk, then it must lie on the boundary of each of the Gergsgorin disks. See [6, Section 10.7, Theorem 2]. So, for $V_{m}$, neither the origin 0 nor $m+1$ can be eigenvalues since they are on the boundary of only two of the Gershgorin disks, not all of them. Thus the inclusion interval given above is, in fact, open and all the eigenvalues of $V_{m}$ are positive.

We repeat our notation from Section 3.1. Throughout this paper, the eigenpairs of $V_{m}$ are denoted $\left(\lambda_{k}, \boldsymbol{z}_{k}\right)$,

$$
\begin{gathered}
0<\lambda_{1}<\lambda_{2}<\cdots<\lambda_{m}<m+1, \\
\boldsymbol{z}_{k}^{t} \boldsymbol{z}_{k}=1, \quad \boldsymbol{z}_{k} \boldsymbol{z}_{j}=0, k \neq j, \quad k=1,2, \ldots, m .
\end{gathered}
$$

The arithmetic mean $\tau$ of the eigenvalues is $\operatorname{trace}\left(V_{m}\right) / m, \tau=\frac{1}{2} m(m+1) / m=\frac{1}{2}(m+1)$. Note that

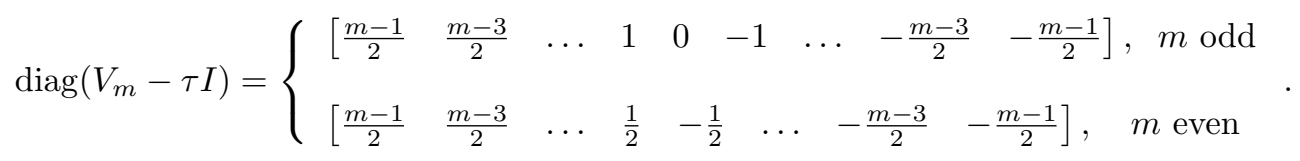

In both cases

$$
S \Varangle\left(V_{m}-\tau I\right) \chi S=-\left(V_{m}-\tau I\right)
$$


which is our substitute for strict skew-persymmetry. So, by Lemma 3.4. $\lambda_{k}$ and $\lambda_{m+1-k}$, for $k=1,2, \ldots,\lfloor m / 2\rfloor$, are equally distant from $\tau$,

$$
\lambda_{m-k+1}-\tau=\tau-\lambda_{k} .
$$

In other words, the eigenvalues are symmetrically arranged around their mean, in pairs.

5.2. Trial eigenpairs. Note that integer $k, k=1, \ldots, m$, is in position $m+1-k$ on $V_{m}$ 's diagonal. We choose to write a typical eigenvector as

$$
\boldsymbol{z}_{k}=\boldsymbol{z}=\left[\begin{array}{lll}
\boldsymbol{x}^{t} & 1 & \boldsymbol{y}^{t}
\end{array}\right]^{t} \alpha
$$

where $\boldsymbol{x} \in \mathbb{R}^{m-k}$ and $\boldsymbol{y} \in \mathbb{R}^{k-1}$. We are interested here in those cases when $\lambda_{k}$ is close to $k$ and this requires $4 \leq k \leq m-3$. In these cases we write

$$
\lambda_{k}=k+\varepsilon
$$

Since $\boldsymbol{z}_{k}$ is defined to have unit 2-norm, then

$$
\alpha=\left(1+\|\boldsymbol{x}\|^{2}+\|\boldsymbol{y}\|^{2}\right)^{-1 / 2} .
$$

We now consider the eigenvector equation $\left(V_{m}-\lambda_{k} I_{m}\right) \boldsymbol{z}=\mathbf{0}$. Unfolding the left-hand side of this equation yields

$$
\begin{aligned}
\left(V_{m}-\lambda_{k} I_{m}\right) \boldsymbol{z}= & {\left[\begin{array}{ccc}
\left(V_{m-k}-\varepsilon I_{m-k}\right) & \boldsymbol{e}_{m-k} & O \\
\boldsymbol{e}_{m-k}^{t} & -\varepsilon & \boldsymbol{e}_{1}^{t} \\
O & \boldsymbol{e}_{1} & -S \Varangle\left(V_{k-1}+\varepsilon I_{k-1}\right) \backslash S
\end{array}\right]\left[\begin{array}{c}
\boldsymbol{x} \\
1 \\
\boldsymbol{y}
\end{array}\right] \alpha } \\
= & {\left[\begin{array}{c}
\left(V_{m-k}-\varepsilon I_{m-k}\right) \boldsymbol{x}+\boldsymbol{e}_{m-k} \cdot 1+0 \cdot \boldsymbol{y} \\
\boldsymbol{e}_{m-k}^{t} \boldsymbol{x}-\varepsilon \cdot 1+\boldsymbol{e}_{1}^{t} \boldsymbol{y} \\
0 \cdot \boldsymbol{x}+\boldsymbol{e}_{1} \cdot 1-S \chi\left(V_{k-1}+\varepsilon I_{k-1}\right) \chi S \boldsymbol{y}
\end{array}\right] \alpha }
\end{aligned}
$$

where $\boldsymbol{e}_{1} \in \mathbb{R}^{k-1}, \quad I=X_{k-1}, S=S_{k-1}$. The first component on the right vanishes when

$$
\boldsymbol{x}=-\left(V_{m-k}-\varepsilon I_{m-k}\right)^{-1} \boldsymbol{e}_{m-k} .
$$

The third component may be rewritten as

$$
\begin{aligned}
\boldsymbol{e}_{1}-S \chi\left(V_{k-1}+\varepsilon I_{k-1}\right) \chi S \boldsymbol{y} & =S \chi\left[\Varangle S \boldsymbol{e}_{1}-\left(V_{k-1}+\varepsilon I_{k-1}\right) \chi S \boldsymbol{y}\right] \\
& =S \chi\left[\boldsymbol{e}_{k-1}-\left(V_{k-1}+\varepsilon I_{k-1}\right) \chi S \boldsymbol{y}\right],
\end{aligned}
$$

which vanishes when

$$
\boldsymbol{y}=S \chi\left(V_{k-1}+\varepsilon I_{k-1}\right)^{-1} \boldsymbol{e}_{k-1} .
$$

On substituting these values of $\boldsymbol{x}$ and $\boldsymbol{y}$, the middle component becomes

$$
\boldsymbol{e}_{m-k}^{t} \boldsymbol{x}-\varepsilon+\boldsymbol{e}_{1}^{t} \boldsymbol{y}=0
$$

or

$$
\varepsilon=-\boldsymbol{e}_{m-k}^{t}\left(V_{m-k}-\varepsilon I_{m-k}\right)^{-1} \boldsymbol{e}_{m-k}+\boldsymbol{e}_{k-1}^{t}\left(V_{k-1}+\varepsilon I_{k-1}\right)^{-1} \boldsymbol{e}_{k-1}
$$


since $\boldsymbol{e}_{1}^{t} S x=\boldsymbol{e}_{k-1}^{t}$.

Equation (5.6) has the form $\varepsilon=f(\varepsilon)$ and could be solved iteratively by $\varepsilon_{j+1}=f\left(\varepsilon_{j}\right), \varepsilon_{0}=0$. Some analysis shows that $f^{\prime}(\varepsilon)=\|\boldsymbol{x}\|^{2}-\|\boldsymbol{y}\|^{2}$ and $\left|f^{\prime}(\varepsilon)\right|<1$ for our values of $k$ and $m \geq 9$. The first iteration gives

$$
\varepsilon_{1}=\delta_{k-1}^{-1}-\delta_{m-k}^{-1}
$$

and the iteration converges rapidly, for our range of $k$ (see $(5.12)$ ).

At this point we consider a new unit length vector $\widetilde{\boldsymbol{z}}$, from $\boldsymbol{z}$ in $(5.2)$, obtained by setting $\varepsilon=0$ in (5.3) and in (5.4). In this case, $\boldsymbol{x}=-V_{m-k}^{-1} \boldsymbol{e}_{m-k}, \boldsymbol{y}=S \chi V_{k-1}^{-1} \boldsymbol{e}_{k-1}$ and

$$
\widetilde{\boldsymbol{z}}=\left[\begin{array}{c}
-V_{m-k}^{-1} \boldsymbol{e}_{m-k} \\
1 \\
S \Varangle V_{k-1}^{-1} \boldsymbol{e}_{k-1}
\end{array}\right] \alpha .
$$

The middle term (5.5) of the eigenvector equation reduces to $\varepsilon_{1}=\delta_{k-1}^{-1}-\delta_{m-k}^{-1}$. This term vanishes only when $k-1=m-k$, that is, when $k=(m+1) / 2$ and $m$ must be odd. This is the only instance when an eigenvalue of $V_{m}$ is an exact integer, $\lambda_{k}=k$. In all other cases

$$
\left\|\left(V_{m}-k I_{m}\right) \widetilde{\boldsymbol{z}}\right\|=\alpha\left|\delta_{k-1}^{-1}-\delta_{m-k}^{-1}\right|>0 .
$$

We now show that $\lambda_{k}$ is an integer to visual accuracy for $4 \leq k \leq m-3$. A basic inequality says that for any conformable vector $\boldsymbol{v} \neq \mathbf{0}$ and non-eigenvalue $\sigma \in \mathbb{R}$ of any real symmetric matrix $M$,

$$
0 \neq\|\boldsymbol{v}\|=\left\|(M-\sigma I)^{-1}(M-\sigma I) \boldsymbol{v}\right\| \leq \frac{1}{\min _{i}\left|\lambda_{i}[M]-\sigma\right|}\|(M-\sigma I) \boldsymbol{v}\| .
$$

By (5.8), for $M=V_{m}, \sigma=k$ and unit lenght $\boldsymbol{v}=\widetilde{\boldsymbol{z}}$, using (5.7) we have

$$
\min _{i}\left|\lambda_{i}\left[V_{m}\right]-k\right|=\left|\lambda_{k}-k\right| \leq\left|\delta_{k-1}^{-1}-\delta_{m-k}^{-1}\right| \alpha=\left|\delta_{m-k}-\delta_{k-1}\right| \frac{\alpha}{\delta_{k-1} \delta_{m-k}} .
$$

Since $\left\{\delta_{m}\right\}$ is monotone decreasing and convergent to $\phi, \delta_{k-1} \delta_{m-k}>\phi^{2}>0.15$. The estimates in Section 4 show that $\|\boldsymbol{x}\|^{2}$ and $\|\boldsymbol{y}\|^{2}$ are close and exceed 8.5 , for all $k-1 \geq 3$ and $m-k \geq 3$, our range of $k$, (see (4.10) and the numerical aproximations below) and so $\alpha<1 / \sqrt{18}$. Hence (5.9) above reduces to the following theorem.

THEOREM 5.1.

$$
\left|\lambda_{k}-k\right|<\frac{\left|\delta_{m-k}-\delta_{k-1}\right|}{0.15 \sqrt{18}}<\frac{8}{5}\left|\delta_{m-k}-\delta_{k-1}\right| .
$$

Now let

$$
l=\min (k-1, m-k)=\left\{\begin{array}{ll}
k-1, & k \leq\lfloor(m+1) / 2\rfloor \\
m-k, & k>\lfloor(m+1) / 2\rfloor
\end{array} .\right.
$$

Writing $\left|\delta_{m-k}-\delta_{k-1}\right|=\left|\delta_{l}-\delta_{l+p}\right|$ for some nonegative integer $p$ and using (4.5) in the proof of Lemma 4.5 with $m=l$, yields

$$
\left|\delta_{m-k}-\delta_{k-1}\right|<\frac{l^{2}}{(l+1)^{2}} \cdot \frac{1}{l !(l-1) !}=\frac{l}{(l+1)^{2}[(l-1) !]^{2}} .
$$


Thus from 5.10 and 5.11 , we obtain

$$
\left|\lambda_{k}-k\right|<\frac{8}{5} \cdot \frac{l}{(l+1)^{2}[(l-1) !]^{2}}, \quad 4 \leq k \leq m-3 .
$$

With $k=5(l=k-1=4)$, for $m \geq 9$,

$$
\left|\lambda_{5}-5\right|<\frac{8}{5} \cdot \frac{4}{5^{2} 3 !^{2}} \simeq 0.0071
$$

well below our level of visual accuracy. So $k$ differs from an eigenvalue by less than the human eye can distinguish. In fact, from numerical computations, at the extremes, $4-\lambda_{4}$ and $\lambda_{m-3}-(m-3)$ are close to 0.004 . Thus, to eyeball accuracy, the eigenvalues of $V_{m}$ are the associated diagonal entries, except for the first three and last three.

We emphasize that the closer $k$ is to $(m+1) / 2$, the closer is $\lambda_{k}$ to $k$. Also, the greater is $m$, the closer is $\lambda_{k}$ to $k$, for $4 \leq k \leq m-3$.

5.3. Exterior eigenpairs. The exterior eigenvalues $\lambda_{1}, \lambda_{2}, \lambda_{3}$ and $\lambda_{m-2}, \lambda_{m-1}, \lambda_{m}$ are of some interest since their separation from the extreme diagonal entries depends weakly on $m$. For $m \geq 7$ computation shows that $\lambda_{1} \simeq 1-0.75, \lambda_{2} \simeq 2-0.2$ and $\lambda_{3} \simeq 3-0.04$.

Since $\lambda_{1}$ is closer to 0 than to 1 , a back-of-the-envelope analysis tests $\boldsymbol{x}=-V_{m}^{-1} \boldsymbol{e}_{m}$ as an approximate eigenvector for $\lambda_{1}$ (approximate eigenvector for $k=0$ ). Its Rayleigh quotient is

$$
\rho:=\frac{\boldsymbol{x}^{t} V_{m} \boldsymbol{x}}{\|\boldsymbol{x}\|^{2}}=\frac{x(m)}{\|\boldsymbol{x}\|^{2}}=\frac{\delta_{m}^{-1}}{\|\boldsymbol{x}\|^{2}} \simeq \frac{\phi^{-1}}{\|\boldsymbol{x}\|^{2}} \simeq 0.271875 \quad\left(\lambda_{1}=0.253806\right) .
$$

This is not a bad approximation to $\lambda_{1}$. Note that our $\boldsymbol{x}$ is the top part of the $\widetilde{z}$ vector in Section 5.2 for $k=0$ (not normalized). The 1 and the $\boldsymbol{y}$ are empty.

In Figure 5.1 we compare the eigenvector $z_{1}$ for $\lambda_{1}$ when $m=15$ with the approximate eigenvectors $\widetilde{\boldsymbol{z}}_{\mathbf{0}}=-V_{m}^{-1} \boldsymbol{e}_{m}$ and $\widetilde{\boldsymbol{z}}_{\mathbf{1}}=\left[\begin{array}{ll}\boldsymbol{x}^{t} & 1\end{array}\right]^{t}, \boldsymbol{x}=-V_{m-1}^{-1} \boldsymbol{e}_{m-1}$ (normalized eigenvectors).

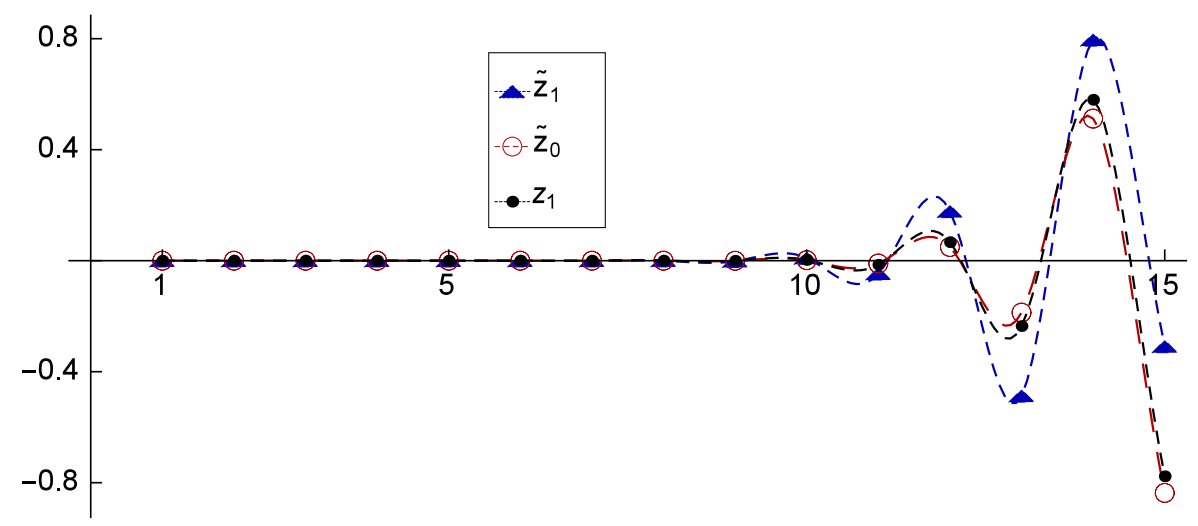

FigURE 5.1. Eigenvector $\boldsymbol{z}_{1}$ of $V_{15}$ for $\lambda_{1} \simeq 0.253806$ and approximate eigenvectors $\widetilde{\boldsymbol{z}}_{\mathbf{0}}$ and $\widetilde{\boldsymbol{z}}_{\mathbf{1}}$.

For the dominant eigenvector (for $\lambda_{m}$ ) it is the top part of $\widetilde{\boldsymbol{z}}$ that is missing and the bottom section $\boldsymbol{y}=S \varkappa \boldsymbol{x}$ that is recognizable. 
Continuing in this vein, we can approximate an eigenvector for an eigenvalue near $k=2,3, m-2, m-1$ by $\widetilde{\boldsymbol{z}}=\left[\begin{array}{lll}\boldsymbol{x}^{t} & 1 & \boldsymbol{y}^{t}\end{array}\right]^{t}$, with $\boldsymbol{x}=-V_{m-k}^{-1} \boldsymbol{e}_{m-k}$ and $\boldsymbol{y}=S \quad V_{k-1}^{-1} \boldsymbol{e}_{k-1}$, whose Rayleigh quotient is

$$
\bar{\rho}:=k+\frac{\delta_{m-k}-\delta_{k-1}}{\delta_{k-1} \delta_{m-k}\|\bar{z}\|^{2}} \simeq k \mp \frac{\delta_{l}-\phi}{\phi \delta_{l}\left(1+\|\boldsymbol{x}\|^{2}+\|\boldsymbol{y}\|^{2}\right)}
$$

with $l=\min (k-1, m-k)$. Observe that since $\left\{\delta_{m}\right\}$ is monotone decreasing and convergent to $\phi$, $\left|\delta_{m-k}-\delta_{k-1}\right|<\left|\delta_{l}-\phi\right|$.

Thus, when $k=2, \boldsymbol{x}=-V_{m-2}^{-1} \boldsymbol{e}_{m-2}, \boldsymbol{y}=[1], \delta_{l}=\delta_{1}=1$, we obtain

$$
\bar{\rho} \simeq 2-\frac{1-0.38821}{0.38821 \cdot(1+9.47467+1)} \simeq 1.86266 \quad\left(\lambda_{2}=1.789321\right),
$$

and when $k=3, \boldsymbol{x}=-V_{m-3}^{-1} \boldsymbol{e}_{m-3}, \boldsymbol{y}=S \Varangle V_{2}^{-1} \boldsymbol{e}_{2}=\left[\begin{array}{ll}-1 & 2\end{array}\right]^{t}, \delta_{l}=\delta_{2}=1 / 2$,

$$
\bar{\rho} \simeq 3-\frac{1 / 2-0.38821}{0.38821 \cdot(1 / 2) \cdot(1+9.47467+5)} \simeq 2.96278 \quad\left(\lambda_{3}=2.961059\right) .
$$

Similarly, for $k=m-2$ and $k=m-1$, we have, respectively,

$$
\bar{\rho} \simeq(m-2)+0.0372173 \quad \text { and } \quad \bar{\rho} \simeq(m-1)+0.137339 .
$$

Thus, when plotted, the eigenvectors for $\lambda_{1}, \lambda_{2}$ and $\lambda_{3}$ are modest distortions of our canonical vector $\boldsymbol{z}$ from 5.2 , forced by a lack of room for the bottom of part $\boldsymbol{y}$. The top section of the eigenvectors is recognizable as $-V_{m-k}^{-1} \boldsymbol{e}_{m-k}(=\boldsymbol{x})$. For the largest three eigenvalues recall, from (5.1), that they are mirror images of the small ones, namely, $\lambda_{m-k+1}=m+1-\lambda_{k}, k=1,2,3$, and it is the bottom section of the eigenvectors that is recognizable as $S \Varangle V_{k-1}^{-1} \boldsymbol{e}_{k-1}(=\boldsymbol{y})$.

6. Secular equations for eigenvalues of $\boldsymbol{W}_{\mathbf{2 m + 1}}^{ \pm}$. All the analysis needed to obtain the eigenvectors of $W_{2 m+1}^{ \pm}$to visual accuracy has been done but before presenting them we wish to share two pictures, Figures 6.1 and 6.2 below, that convey all needed information concerning the eigenvalues.

6.1. $\boldsymbol{W}_{\mathbf{2} \boldsymbol{m}+\mathbf{1}}^{-}$. We take trial vector $\boldsymbol{w}=\left[\begin{array}{lll}\boldsymbol{u}^{t} & 1 & \boldsymbol{v}^{t}\end{array}\right]^{t}$ for $\boldsymbol{u} \in \mathbb{R}^{m}, \boldsymbol{v} \in \mathbb{R}^{m}$, a typical eigenvalue $\mu$ and examine the components of $\left(W_{2 m+1}^{-}-\mu I_{2 m+1}\right) \boldsymbol{w}$. Use 3.1 to find

$$
\left(W_{2 m+1}^{-}-\mu I_{2 m+1}\right)\left[\begin{array}{l}
\boldsymbol{u} \\
1 \\
\boldsymbol{v}
\end{array}\right]=\left[\begin{array}{c}
\left(V_{m}-\mu I_{m}\right) \boldsymbol{u}+\boldsymbol{e}_{m} \cdot 1+\mathbf{0} \\
\boldsymbol{e}_{m}^{t} \boldsymbol{u}-\mu \cdot 1+\boldsymbol{e}_{1}^{t} \boldsymbol{v} \\
\mathbf{0}+\boldsymbol{e}_{1} \cdot 1-\chi S\left(V_{m}+\mu I_{m}\right) S \Varangle \boldsymbol{v}
\end{array}\right]
$$

The right choice for $\boldsymbol{u}$ is $\boldsymbol{u}=-\left(V_{m}-\mu I_{m}\right)^{-1} \boldsymbol{e}_{m}$ and the right choice for $\boldsymbol{v}$ is

$$
\boldsymbol{v}=\chi S\left(V_{m}+\mu I_{m}\right)^{-1} S \Varangle \boldsymbol{e}_{1}=\chi S\left(V_{m}+\mu I_{m}\right)^{-1} S \boldsymbol{e}_{m}=S \chi\left(V_{m}+\mu I_{m}\right)^{-1} \boldsymbol{e}_{m},
$$

since $S \boldsymbol{e}_{m}=\boldsymbol{e}_{m}, X S=S X$, when $m$ is odd, and $S \boldsymbol{e}_{m}=-\boldsymbol{e}_{m},-X S=S X$, when $m$ is even.

With these choices the middle component turns out to be

$$
\begin{array}{r}
-\boldsymbol{e}_{m}^{t}\left(V_{m}-\mu I_{m}\right)^{-1} \boldsymbol{e}_{m}-\mu+\boldsymbol{e}_{1}^{t} S \chi\left(V_{m}+\mu I_{m}\right)^{-1} \boldsymbol{e}_{m}= \\
=-\boldsymbol{e}_{m}^{t}\left(V_{m}-\mu I_{m}\right)^{-1} \boldsymbol{e}_{m}-\mu+\boldsymbol{e}_{m}^{t}\left(V_{m}+\mu I_{m}\right)^{-1} \boldsymbol{e}_{m} .
\end{array}
$$


Now it is time to use the spectral factorization of $V_{m}, V_{m}=Z \Lambda Z^{t}, I=Z Z^{t}$, to see that $\mu$ is an eigenvalue of $W_{2 m+1}^{-}$if, and only if,

$$
\mu=-\sum_{k=1}^{m} \frac{\boldsymbol{z}_{k}(m)^{2}}{\lambda_{k}-\mu}+\sum_{k=1}^{m} \frac{\boldsymbol{z}_{k}(m)^{2}}{\lambda_{k}+\mu} .
$$

Note first that $\mu=0$ is one solution. Next divide through by $\mu \neq 0$ and combine the two sums to find our desired secular (= rational) equation for the nonzero eigenvalues of $W_{2 m+1}^{-}$:

$$
\frac{1}{2}=\sum_{k=1}^{m} \frac{\boldsymbol{z}_{k}(m)^{2}}{\mu^{2}-\lambda_{k}^{2}}
$$

The right hand side of 6.1 is an even function of $\mu$ and thus the solutions occur in \pm pairs. It has $2 m$ distinct simple poles at $\pm \lambda_{k}, k=1, \ldots, m$. When $\mu>0$, it has negative slope and approaches 0 as $\mu \longrightarrow+\infty$. Thus the positive solutions $\lambda_{k}^{-}$are to the right of the poles. Let us label the solutions of 6.1) as $\pm \lambda_{k}^{-}$and conclude that

$$
\left( \pm \lambda_{k}^{-}\right)^{2}>\lambda_{k}^{2}>0, \quad i=1,2, \ldots, m .
$$

The zero eigenvalue must have multiplicity $1(=2 m+1-2 m)$. We exhibit its eigenvector $\boldsymbol{w}_{0}$ in Section 7.1. See also Figure 1.2 in the Introduction.

Figure 6.1 shows, for $m=15$, the graphs of $y=\sum_{k=1}^{m} \frac{\boldsymbol{z}_{k}(m)^{2}}{\mu^{2}-\lambda_{k}^{2}}$ and $y=\frac{1}{2}$, for $\mu>0$.

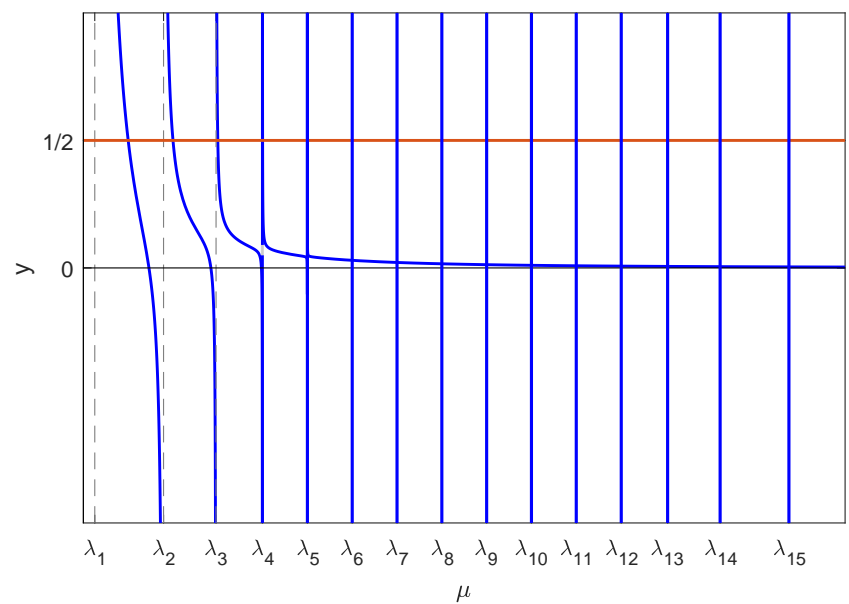

Figure 6.1. Solutions $\mu>0$ of the equation $\sum_{k=1}^{m} \frac{\boldsymbol{z}_{k}(m)^{2}}{\mu^{2}-\lambda_{k}^{2}}=\frac{1}{2}$ for $m=15$.

6.2. $\boldsymbol{W}_{\mathbf{2} \boldsymbol{m + 1}}^{+}$. This matrix is persymmetric $\left(X W_{2 m+1}^{+} \mathbb{X}=W_{2 m+1}^{+}\right)$and its eigenvectors are alternately symmetric and anti-symmetric about the middle entry (index $m+1$ ). The eigenpairs of the smaller matrix $V_{m}$, the leading principal submatrix of $W_{2 m+1}^{+}$, play a crucial role in describing the eigenpairs of $W_{2 m+1}^{+}$. 
For $k=1,2, \ldots, m$, with $V_{m} \boldsymbol{z}_{k}=\boldsymbol{z}_{k} \lambda_{k}$, use (3.1) to verify that the anti-symmetric vector with respect to the middle entry $\boldsymbol{w}=\left[\begin{array}{lll}\boldsymbol{z}_{k}^{t} & 0 & -\boldsymbol{z}_{k}^{t} \boldsymbol{X}\end{array}\right]^{t}$ is an eigenvector of $W_{2 m+1}^{+}$with eigenvalue $\lambda_{k}$,

$$
W_{2 m+1}^{+}\left[\begin{array}{c}
\boldsymbol{z}_{k} \\
0 \\
-\chi \boldsymbol{z}_{k}
\end{array}\right]=\left[\begin{array}{c}
V_{m} \boldsymbol{z}_{k}+\boldsymbol{e}_{m} \cdot 0+\mathbf{0} \\
\boldsymbol{e}_{m}^{t} \boldsymbol{z}_{k}+0-\boldsymbol{e}_{1}^{t} \boldsymbol{X} \boldsymbol{z}_{k} \\
\mathbf{0}+\boldsymbol{e}_{1} \cdot 0-\Varangle V_{m} \chi^{2} \boldsymbol{z}_{k}
\end{array}\right]=\left[\begin{array}{c}
\boldsymbol{z}_{k} \\
0 \\
-\chi \boldsymbol{z}_{k}
\end{array}\right] \lambda_{k} .
$$

This accounts for $m$ eigenpairs and leaves $m+1$ to find. These we denote by $\lambda_{k}^{+}$for $k=0,1,2, \ldots, m$. Our symmetric trial eigenvector has the form $\boldsymbol{w}^{+}=\left[\begin{array}{lll}\boldsymbol{u}^{t} & 1 & \boldsymbol{u}^{t} X\end{array}\right]^{t}$ and when one examines the three components of $\left(W_{2 m+1}^{+}-\mu I_{2 m+1}\right) \boldsymbol{w}^{+}$one finds

$$
\left(W_{2 m+1}^{+}-\mu I_{2 m+1}\right)\left[\begin{array}{c}
\boldsymbol{u} \\
1 \\
\chi \boldsymbol{u}
\end{array}\right]=\left[\begin{array}{c}
\left(V_{m}-\mu I_{m}\right) \boldsymbol{u}+\boldsymbol{e}_{m} \\
\boldsymbol{e}_{m}^{t} \boldsymbol{u}-\mu+\boldsymbol{e}_{1}^{t} \chi \boldsymbol{u} \\
\boldsymbol{e}_{1}+\left(\chi V_{m} X-\mu I_{m}\right)(X \boldsymbol{u})
\end{array}\right]=\left[\begin{array}{c}
\left(V_{m}-\mu I_{m}\right) \boldsymbol{u}+\boldsymbol{e}_{m} \\
2 \boldsymbol{e}_{m}^{t} \boldsymbol{u}-\mu \\
\chi_{m}\left[\left(V_{m}-\mu I_{m}\right) \boldsymbol{u}+\boldsymbol{e}_{m}\right]
\end{array}\right]
$$

It turns out that the right choice for $\boldsymbol{u}$ is $\boldsymbol{u}=-\left(V_{m}-\mu I_{m}\right)^{-1} \boldsymbol{e}_{m}$ and, using this value, the middle component gives the needed equation

$$
-\mu-2 \boldsymbol{e}_{m}^{t}\left(V_{m}-\mu I_{m}\right)^{-1} \boldsymbol{e}_{m}=0 .
$$

Here is the place to explain Wilkinson's use of an auxiliary unsymmetric $(m+1) \times(m+1)$ matrix $U_{m+1}$, along with $V_{m}$, to give the eigenvalues of $W_{2 m+1}^{+}$. See [15, p. 309]. The last pivot of the triangular factorization of the leading principal $(m+1) \times(m+1)$ submatrix of $W_{2 m+1}^{+}-\mu I_{2 m+1}$, namely, $\left[\begin{array}{cc}V_{m}-\mu I_{m} & \boldsymbol{e}_{m} \\ \boldsymbol{e}_{m}^{t} & -\mu\end{array}\right]$, is

$$
-\mu-\boldsymbol{e}_{m}^{t}\left(V_{m}-\mu I_{m}\right)^{-1} \boldsymbol{e}_{m}
$$

which is the expression 6.2 derived above except for the factor 2. So, Wilkinson just changed the $(m+1, m)$ entry of this submatrix to 2 and he had a simple matrix

$$
U_{m+1}=\left[\begin{array}{cc}
V_{m} & \boldsymbol{e}_{m} \\
2 \boldsymbol{e}_{m}^{t} & 0
\end{array}\right]
$$

that gave the needed $m+1$ eigenvalues of $W_{2 m+1}^{+}$. In contrast, our derivation 6.2 above reveals in a natural way the contribution to the middle component from the submatrix $\chi V_{m} \chi$ below it. No need for $U_{m+1}$.

Invoking $V_{m}$ 's spectral factorization in 6.2 gives our desired secular equation

$$
\frac{1}{2} \mu=\sum_{k=1}^{m} \frac{\boldsymbol{z}_{k}(m)^{2}}{\mu-\lambda_{k}} .
$$

The right hand side has poles at the $\lambda_{k}$, goes to 0 as $\mu \longrightarrow \pm \infty$, and has negative slope elsewhere. The left hand side is a straight line passing through 0 and so there is just one negative solution $\mu$ which we label $\lambda_{0}^{+}$while all other solutions $\lambda_{k}^{+}$satisfy $\lambda_{k}^{+}>\lambda_{k}$, for $k=1,2, \ldots, m$. Since the slope of the left hand side of 6.3$)$ is $\frac{1}{2}$, we see that $\lambda_{k}^{+}-\lambda_{k}$ diminishes steadly as $k$ increases and, when $m=15, \lambda_{11}^{+}-\lambda_{11} \simeq 10^{-14}$. The steady variation in $\lambda_{k}^{+}-\lambda_{k}$ makes $W_{2 m+1}^{+}$a demanding test matrix for eigenvalue software.

Figure 6.2 shows the graphs of $y=\sum_{k=1}^{m} \frac{\boldsymbol{z}_{k}(m)^{2}}{\mu-\lambda_{k}}$ and $y=\frac{1}{2} \mu$ for $m=15$. Zooming in this figure we obtain Figure 6.3, for $\lambda_{11}^{+}$. 


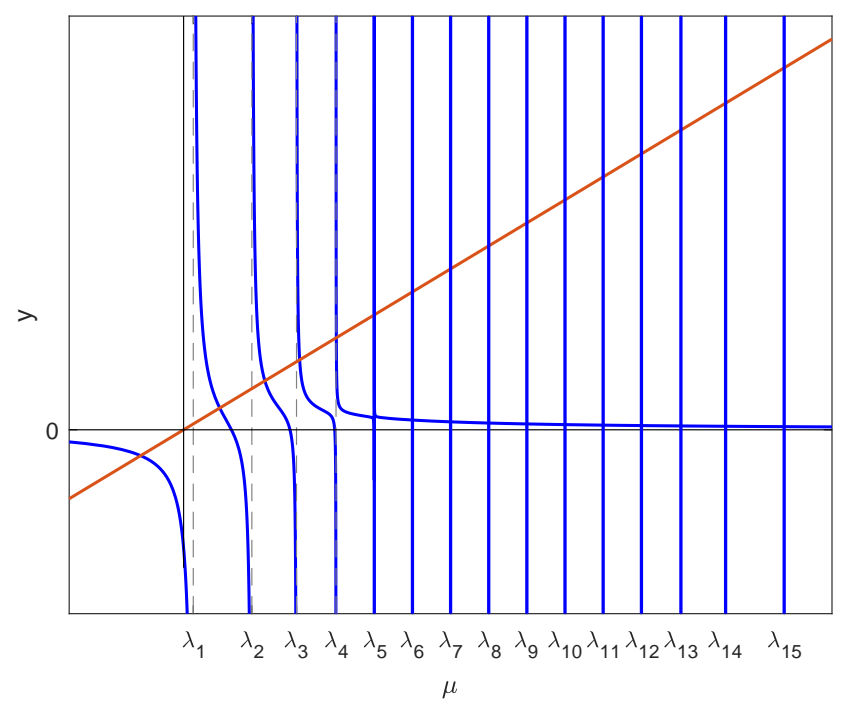

Figure 6.2. Solutions $\mu$ of the equation $\sum_{k=1}^{m} \frac{\boldsymbol{z}_{k}(m)^{2}}{\mu-\lambda_{k}}=\frac{1}{2} \mu$ for $m=15$.

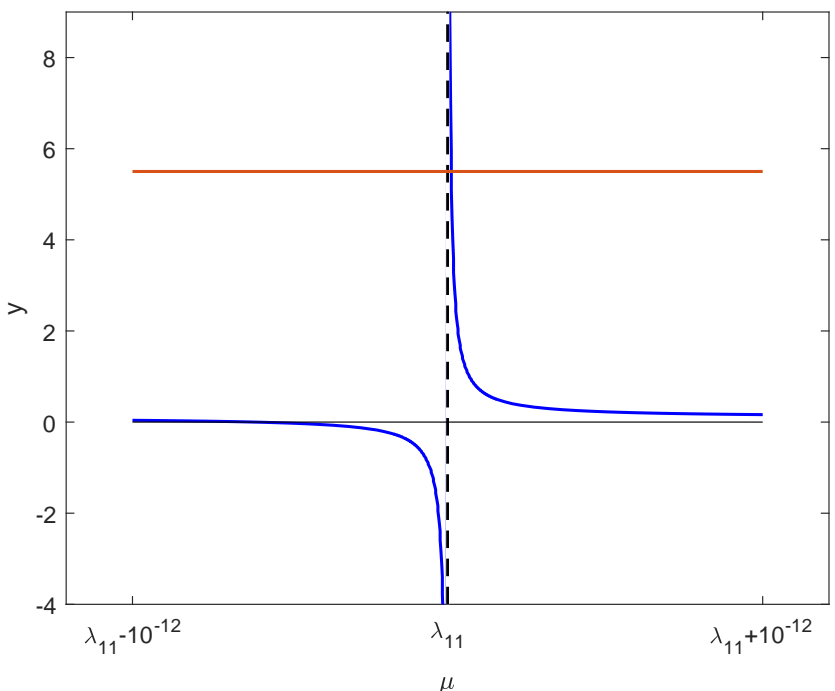

Figure 6.3. Eigenvalue $\lambda_{11}^{+}$of $W_{31}^{+}$with $\left|\lambda_{11}^{+}-\lambda_{11}\right| \simeq 10^{-14}$. The slope of the "horizontal" line is $1 / 2$.

\section{Eigenvectors for near-integer eigenvalues of $W_{2 m+1}^{ \pm}$.}

7.1. $\boldsymbol{W}_{\mathbf{2 m + 1}}^{-}$. Recall from the previous section that the eigenvalues of $W_{2 m+1}^{-}$, solutions of the equation 6.1], include 0 , are well separated and occur in \pm pairs, labeled $\pm \lambda_{k}^{-}$. The absolute values 
$\lambda_{k}^{-}$of the eigenvalues satisfy

$$
\lambda_{0}^{-}=0<\lambda_{1}<\lambda_{1}^{-}<\lambda_{2}<\lambda_{2}^{-}<\cdots<\lambda_{m}<\lambda_{m}^{-}
$$

where $\lambda_{k}$ is an eigenvalue of $V_{m}, k=1, \ldots, m$.

Since $W_{2 m+1}^{-}=V_{2 m+1}-(m+1) I_{2 m+1}$ the eigenvectors of $W_{2 m+1}^{-}$are the same as those of $V_{2 m+1}$ and these were presented in Section 5.2. We content ourselves with a few observations. Since $S \& W_{2 m+1}^{-} \backslash S=-W_{2 m+1}^{-}$it follows that the eigenvector for the eigenvalue (close to) $-k$ $(0 \leq k \leq m-3)$ is obtained by applying $X S$ to the eigenvector for the eigenvalue (close to) $k$. See Lemma 3.4 . The envelopes look the same but one has its middle entry at $m+1+k$, the other at $m+1-k$.

The middle eigenvalue of $V_{2 m+1}$ is $m+1$ and the eigenvector for $\lambda_{0}^{-}=(m+1)-(m+1)=0$ of $W_{2 m+1}^{-}$is the only one for which both $\boldsymbol{x}$ and $\boldsymbol{y}$ in $\widetilde{\boldsymbol{z}}$ in Section 5.2 belong to $\mathbb{R}^{m}$, since, in that context,

$$
(2 m+1)-(m+1)=(m+1)-1=m .
$$

So eigenvector $\boldsymbol{w}_{0} \simeq \widetilde{\boldsymbol{z}} / \alpha$ for eigenvalue 0 has a top part $\boldsymbol{x}$ and a bottom part $\boldsymbol{y}=-S \nvdash \boldsymbol{x}$,

$$
\boldsymbol{w}_{0} \simeq\left[\begin{array}{l}
\boldsymbol{x} \\
1 \\
\boldsymbol{y}
\end{array}\right]=\left[\begin{array}{c}
-V_{m}^{-1} \boldsymbol{e}_{m} \\
1 \\
S \Varangle V_{m}^{-1} \boldsymbol{e}_{m}
\end{array}\right]
$$

In Section 4.1 we saw that the entries in $V_{m}^{-1} \boldsymbol{e}_{m}$ alternate in sign and that the last entry is equal to $\delta_{m}^{-1}$ and it is always positive. It turns out that only the last 4 entries in $V_{m}^{-1} \boldsymbol{e}_{m} /\left\|V_{m}^{-1} \boldsymbol{e}_{m}\right\|$ exceed $1 / 100$ in magnitude for all $m \geq 4$. Thus, thanks to $X$, the eigenvector $\boldsymbol{w}_{0}$ has its visual support on the 9 indices $m+1-4: m+1+4$.

Consider an eigenvalue of $W_{2 m+1}^{-}$close to integer $k, 0<k \leq m-3$. Observe that $k=(m+1+k)-(m+1)$ and it is easy to verify that an approximate eigenvector $\widetilde{\boldsymbol{w}}$ for $k$ has a top part $\boldsymbol{x}=-V_{m-k}^{-1} \boldsymbol{e}_{m-k} \in \mathbb{R}^{m-k}$ and a bottom part $\boldsymbol{y}=S \nmid V_{m+k}^{-1} \boldsymbol{e}_{m+k} \in \mathbb{R}^{m+k}$. In the context of Section 5.2 for $V_{2 m+1}$ we take the eigenvalue close to $m+1+k$ and

$$
(2 m+1)-(m+1+k)=m-k, \quad(m+1+k)-1=m+k .
$$

We emphasize that for $m-k \geq 4$ both $|\boldsymbol{x}|$ and $|\boldsymbol{y}|$ above look like $\left|V_{m}^{-1} \boldsymbol{e}_{m}\right|$ provided $m \geq 4$. Thus, as $k$ varies the envelope of the associated eigenvector looks just like the envelope of the eigenvector $\boldsymbol{w}_{0}$ for eigenvalue 0 but with its middle entry at index $m+1-k$.

Figure 7.1 shows the eigenvectors described above for eigenvalues close to $k= \pm 11$ when $m=15$. See Figure 1.2 in the Introduction for the characteristic envelopes (dented bell curves) of these eigenvectors. See also Figure 7.2 which shows the envelopes of eigenvectors for eigenvalues close to $k=6,7,8$.

The largest three eigenvalues in magnitude, approximately $\pm(m+3 / 4), \pm[(m-1)+1 / 5]$, $\pm[(m-2)+1 / 25]$, are not close to integers and their eigenvectors do not have room for the characteristic 9-index envelope (see Figure 1.1); they are different from those above only in their lack of symmetry about a central entry. For example, the eigenvector associated with $+(m+3 / 4)$ is close to $S \chi V_{2 m+1}^{-1} e_{2 m+1}$ and its envelope is close to the lower part of the envelope of $W_{2 m+1}^{-}$'s eigenvector $\boldsymbol{w}_{0}$. The envelope of the eigenvector associated with $-(m+3 / 4)$ is $-V_{2 m+1}^{-1} \boldsymbol{e}_{2 m+1}$ which is very close to the top portion of the envelope of the eigenvector $\boldsymbol{w}_{0}$. In Figure 7.3 we exhibit the envelopes of the eigenvectors for the largest three eigenvalues of $W_{31}^{-}$. 


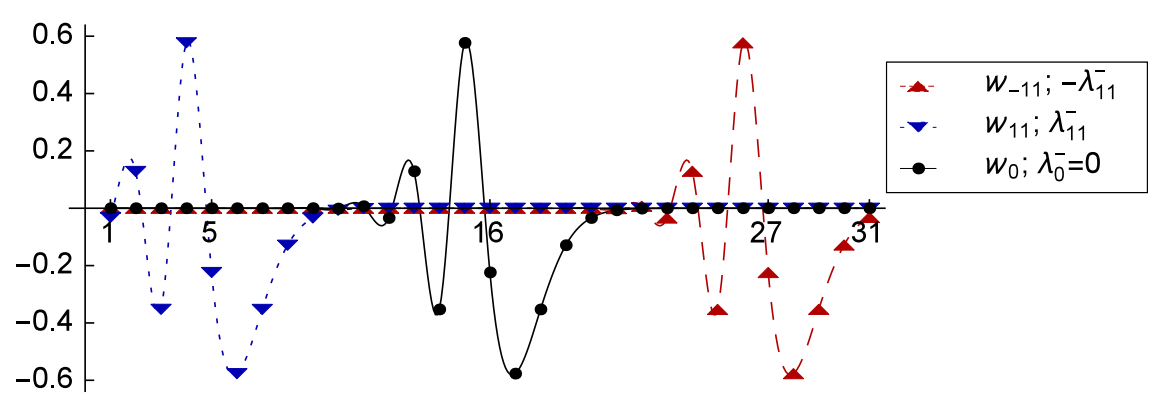

Figure 7.1. Eigenvectors $\boldsymbol{w}_{0}, \boldsymbol{w}_{-11}$ and $\boldsymbol{w}_{11}$ of $W_{31}^{-}$for eigenvalues $0,-\lambda_{11}^{-}$and $\lambda_{11}^{-}$, respectively.

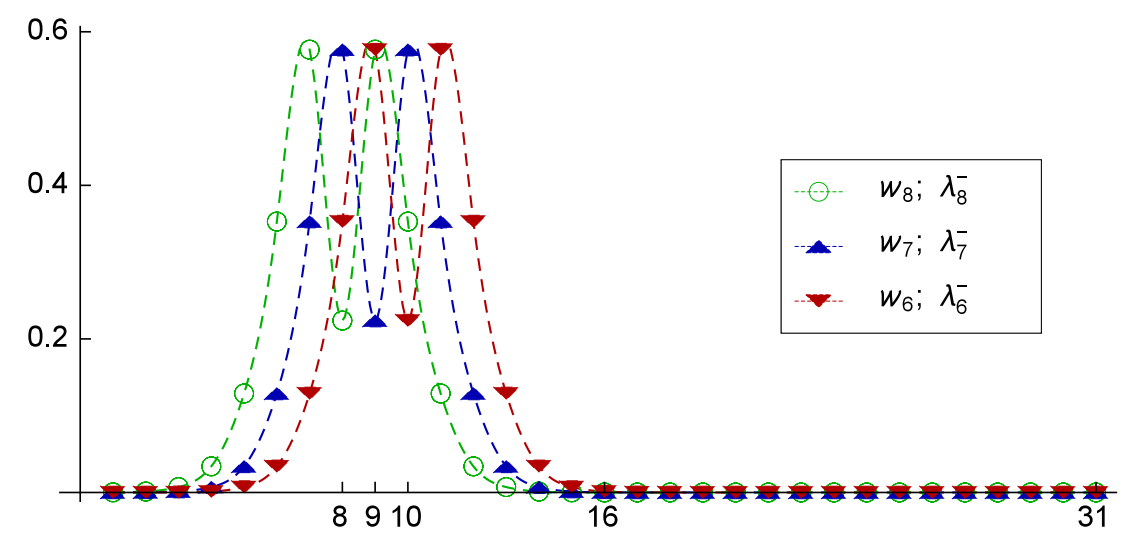

Figure 7.2. $W_{31}^{-}$envelopes of eigenvectors $\boldsymbol{w}_{k}, k=6,7,8$, for $\lambda_{k}^{-}$, respectively.

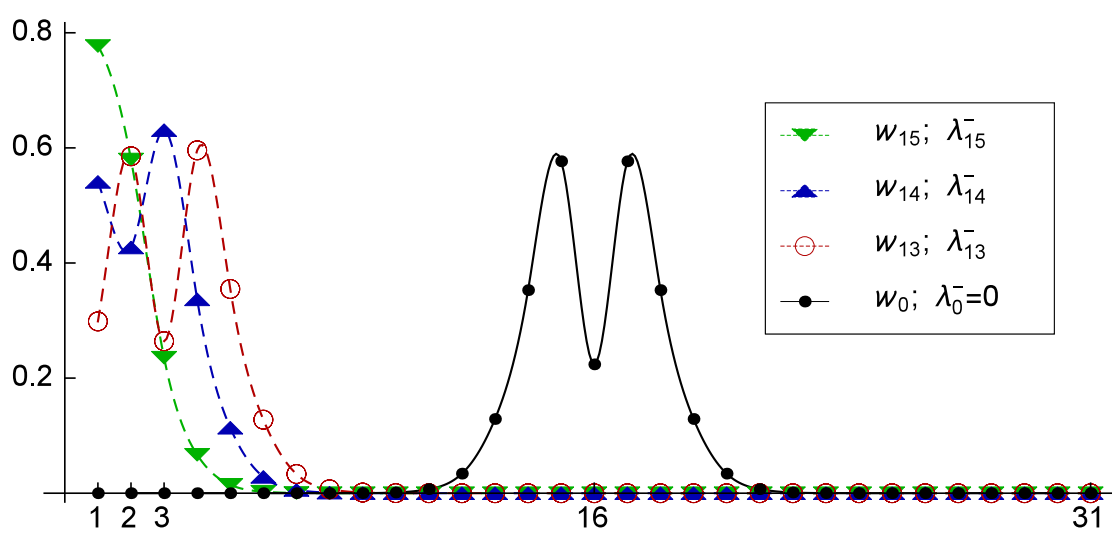

Figure 7.3. Envelopes of $W_{31}^{-}$eigenvectors for eigenvalues $\lambda_{0}^{-}, \lambda_{13}^{-}, \lambda_{14}^{-}$and $\lambda_{15}^{-}$.

7.2. $\boldsymbol{W}_{2 m+1}^{+}$. These eigenvectors are more complicated than those of $W_{2 m+1}^{-}$. From Section 6.2 each eigenvalue $\lambda_{k}$ of $V_{m}$ is an eigenvalue of $W_{2 m+1}^{+}$and the other $m+1$ eigenvalues are solutions 
of equation (6.3). All together these eigenvalues satisfy

$$
\lambda_{0}^{+}<0<\lambda_{1}<\lambda_{1}^{+}<\lambda_{2}<\lambda_{2}^{+}<\cdots<\lambda_{m}<\lambda_{m}^{+}
$$

and, apart from $\lambda_{0}^{+}$and the four positive eigenvalues closest to 0 , all the others occur in pairs $\lambda_{k}, \lambda_{k}^{+}$ getting closer and closer to each other as $k$ increases. This is independent of whether $\lambda_{k}$ is close to an integer. We acknowledge that $\lambda_{0}^{+}$is a strange designation for a negative number but we feel that our simple labels for the whole set of $m+1$ solutions to equation 6.3 is adequate compensation. Furthermore for $4 \leq k \leq m-3$ both $\lambda_{k}$ and $\lambda_{k}^{+}$are visually close to $k$ and

$$
\begin{array}{ll}
\lambda_{k}<k<\lambda_{k}^{+}, & \text {for } k<(m+1) / 2, \\
k \leq \lambda_{k}<\lambda_{k}^{+}, & \text {for } k \geq(m+1) / 2 .
\end{array}
$$

For our range $4 \leq k \leq m-3$ these envelopes look alike. Our goal is to see why this occurs and identify its limitations for extreme values of $k$.

By persymetry of $W_{2 m+1}^{+}$, the eigenvector $\boldsymbol{w}$ of $\lambda_{k}$ satisfies $X_{2 m+1} \boldsymbol{w}=-\boldsymbol{w}$, while the eigenvector $\boldsymbol{w}^{+}$for $\lambda_{k}^{+}$satisfies $X_{2 m+1} \boldsymbol{w}^{+}=\boldsymbol{w}^{+}$. Our notation is

$$
\boldsymbol{w}:=\left[\begin{array}{lll}
\boldsymbol{z}^{t} & 0 & -\boldsymbol{z}^{t} 1_{m}
\end{array}\right]^{t}, \quad \boldsymbol{w}^{+}:=\left[\begin{array}{lll}
\boldsymbol{u}^{t} & \beta & \boldsymbol{u}^{t} 1_{m}
\end{array}\right]^{t}, \quad\|\boldsymbol{u}\|=1, \quad \beta \neq 0 .
$$

Below we will compare the top part $\boldsymbol{u}\left(=\boldsymbol{u}_{k}\right)$ of $\boldsymbol{w}^{+}$to the top part $\boldsymbol{z}\left(=\boldsymbol{z}_{k}\right)$ of $\boldsymbol{w}$.

We now embark on the most complicated analyses in the paper all aimed at producing the comparisons shown in 7.13 . It is most convenient to proceed in two stages. We write

$$
\lambda_{k}^{+}=k+\eta
$$

where $\eta$ is small but not 0 . The analysis in Section 6.2 gives $\left(W_{2 m+1}^{+}-\lambda_{k}^{+} I_{2 m+1}\right) \boldsymbol{w}^{+}$in three parts as

$$
\begin{aligned}
& \left(V_{m}-\lambda_{k}^{+} I_{m}\right) \boldsymbol{u}+\boldsymbol{e}_{m} \beta \\
& \boldsymbol{e}_{m}^{t} \boldsymbol{u}-\lambda_{k}^{+} \beta+\boldsymbol{e}_{1}^{t} \Varangle \boldsymbol{u} \\
& \boldsymbol{e}_{1} \beta+\left(\Varangle V_{m} X-\lambda_{k}^{+} I_{m}\right) \Varangle \boldsymbol{u}=\Varangle\left[\Varangle \boldsymbol{e}_{1} \beta+\left(V_{m}-\lambda_{k}^{+} I_{m}\right) \boldsymbol{u}\right] .
\end{aligned}
$$

Both (7.2) and (7.4) vanish when $\boldsymbol{u}=-\left(V_{m}-\lambda_{k}^{+} I_{m}\right)^{-1} \boldsymbol{e}_{m} \beta$ while 7.3 evaluates to

$$
\gamma:=2 \boldsymbol{e}_{m}^{t} \boldsymbol{u}-\lambda_{k}^{+} \beta=-\beta\left[2 \boldsymbol{e}_{m}^{t}\left(V_{m}-\lambda_{k}^{+} I_{m}\right)^{-1} \boldsymbol{e}_{m}+\lambda_{k}^{+}\right]
$$

and must also vanish.

Note that $\operatorname{diag}\left(V_{m}-k I_{m}\right)=\left[\begin{array}{lllllll}m-k & \ldots & 1 & 0 & -1 & \ldots & -k+1\end{array}\right]$ which suggests our notation for the top part of $\boldsymbol{w}^{+}$,

$$
\boldsymbol{u}=\left[\begin{array}{lll}
\overline{\boldsymbol{x}}^{t} & 1 & \overline{\boldsymbol{y}}^{t}
\end{array}\right]^{t} \bar{\alpha}, \quad \overline{\boldsymbol{x}} \in \mathbb{R}^{m-k}, \quad \overline{\boldsymbol{y}} \in \mathbb{R}^{k-1}, \quad \bar{\alpha}=\left(1+\|\overline{\boldsymbol{x}}\|^{2}+\|\overline{\boldsymbol{y}}\|^{2}\right)^{-1 / 2} .
$$

Now unpack (7.2)- (7.4) using $\lambda_{k}^{+}=k+\eta$, to obtain

$$
\begin{aligned}
\bar{\alpha}\left[\left(V_{m-k}-\eta I_{m-k}\right) \overline{\boldsymbol{x}}+\boldsymbol{e}_{m-k}\right] & =0 \\
\bar{\alpha}\left(\boldsymbol{e}_{m-k}^{t} \overline{\boldsymbol{x}}-\eta \cdot 1+\boldsymbol{e}_{1}^{t} \overline{\boldsymbol{y}}\right) & =0 \\
\bar{\alpha}\left\{\boldsymbol{e}_{1}-\left[S \Psi\left(V_{k-1}+\eta I_{k-1}\right) \backslash S\right] \overline{\boldsymbol{y}}\right\}=S X\left[X S \boldsymbol{e}_{1}-\left(V_{k-1}+\eta I_{k-1}\right) \chi S \overline{\boldsymbol{y}}\right] \bar{\alpha} & =-\boldsymbol{e}_{k-1} \beta .
\end{aligned}
$$


Note that 7.7 is not homogeneous. Section 7.3 shows that $\eta$ is small enough that $V_{m-k}-\eta I_{m-k}$ is still positive definite. From 7.5 we obtain

$$
\overline{\boldsymbol{x}}=-\left(V_{m-k}-\eta I_{m-k}\right)^{-1} \boldsymbol{e}_{m-k} .
$$

Next rewrite (7.7) as

$$
\left(V_{k-1}+\eta I_{k-1}\right) \chi S \overline{\boldsymbol{y}}=\Varangle S \boldsymbol{e}_{1}+\Varangle S \boldsymbol{e}_{k-1} \beta / \bar{\alpha} .
$$

Recall that

$$
\Psi S \boldsymbol{e}_{k-1}=\left\{\begin{aligned}
\boldsymbol{e}_{1}, & k \text { even }, \\
-\boldsymbol{e}_{1}, & k \text { odd }
\end{aligned}\right.
$$

while $X S \boldsymbol{e}_{1}=\boldsymbol{e}_{k-1}$ always. Thus

$$
\overline{\boldsymbol{y}}=S X\left(V_{k-1}+\eta I_{k-1}\right)^{-1}\left(\boldsymbol{e}_{k-1} \pm \boldsymbol{e}_{1} \beta / \bar{\alpha}\right) .
$$

Constraint (7.6) becomes

$$
-\boldsymbol{e}_{m-k}^{t}\left(V_{m-k}-\eta I_{m-k}\right)^{-1} \boldsymbol{e}_{m-k}-\eta+\boldsymbol{e}_{k-1}^{t}\left(V_{k-1}+\eta I_{k-1}\right)^{-1}\left(\boldsymbol{e}_{k-1} \pm \boldsymbol{e}_{1} \beta / \bar{\alpha}\right)=0
$$

and observe that for small enough $\eta(k \geq 4)$ it reduces to

$$
-\boldsymbol{e}_{m-k}^{t} V_{m-k}^{-1} \boldsymbol{e}_{m-k}-\eta+\boldsymbol{e}_{k-1}^{t} V_{k-1}^{-1}\left(\boldsymbol{e}_{k-1} \pm \boldsymbol{e}_{1} \beta / \bar{\alpha}\right) \simeq 0
$$

or

$$
\left(\delta_{k-1}^{-1}-\delta_{m-k}^{-1}\right) \bar{\alpha}-\eta \bar{\alpha} \pm \boldsymbol{e}_{k-1}^{t} V_{k-1}^{-1} \boldsymbol{e}_{1} \beta \simeq 0 .
$$

Note that (7.11) is an approximation to the exact 7.9 and both bring a new quantity into the analysis. By symmetry of $V_{k-1}$,

$$
\omega=\omega_{k-1}:=\boldsymbol{e}_{k-1}^{t} V_{k-1}^{-1} \boldsymbol{e}_{1}=\boldsymbol{e}_{1}^{t} V_{k-1}^{-1} \boldsymbol{e}_{k-1},
$$

and so $\omega$ is the top entry of $V_{k-1}^{-1} e_{k-1} \in \mathbb{R}^{k-1}$. By Lemma 4.6

$$
|\omega|<\frac{1}{\phi(k-2) !}<\frac{3}{(k-2) !}
$$

which shows why we do not consider small values of $k(k=2,3)$ in what follows.

When $m$ is odd then $k=(m+1) / 2$ is an integer eigenvalue of both $V_{m}$ and $W_{2 m+1}^{+}$. Note that 7.5. determines $\overline{\boldsymbol{x}}$ independently of $\beta$.

At this point we begin stage 2 . We are ready to compare the eigenvectors $\boldsymbol{w}$ for $\lambda_{k}=k+\varepsilon$ and 
$\boldsymbol{w}^{+}$for $\lambda_{k}^{+}=k+\eta$ in our range $4 \leq k \leq m-3$ :

$$
\boldsymbol{w}=\left[\begin{array}{c}
\boldsymbol{z}_{k} \\
0 \\
-1 \boldsymbol{z}_{k}
\end{array}\right], \quad \boldsymbol{w}^{+}=\left[\begin{array}{c}
\boldsymbol{u}_{k} \\
\beta \\
\Varangle \boldsymbol{u}_{k}
\end{array}\right], \quad \boldsymbol{u}_{k}=-\left(V_{m}-\lambda_{k}^{+} I_{m}\right)^{-1} \boldsymbol{e}_{m} \beta,
$$

where

$$
\begin{aligned}
\boldsymbol{z}_{k} & =\left[\begin{array}{l}
\boldsymbol{x} \\
1 \\
\boldsymbol{y}
\end{array}\right] \alpha \quad(\text { Section } \underline{5.2}) & \boldsymbol{u}_{k} & =\left[\begin{array}{c}
\overline{\boldsymbol{x}} \\
1 \\
\overline{\boldsymbol{y}}
\end{array}\right] \bar{\alpha} \\
\alpha & =\left(1+\|\boldsymbol{x}\|^{2}+\|\boldsymbol{y}\|^{2}\right)^{-1 / 2} & \bar{\alpha} & =\left(1+\|\overline{\boldsymbol{x}}\|^{2}+\|\overline{\boldsymbol{y}}\|^{2}\right)^{-1 / 2} \\
\boldsymbol{x} & =-\left(V_{m-k}-\varepsilon I_{m-k}\right)^{-1} \boldsymbol{e}_{m-k} & \overline{\boldsymbol{x}} & =-\left(V_{m-k}-\eta I_{m-k}\right)^{-1} \boldsymbol{e}_{m-k} \\
\boldsymbol{y} & =S X\left(V_{k-1}+\varepsilon I_{k-1}\right)^{-1} \boldsymbol{e}_{k-1} & \overline{\boldsymbol{y}} & =S X\left(V_{k-1}+\eta I_{k-1}\right)^{-1}\left(\boldsymbol{e}_{k-1} \pm \boldsymbol{e}_{1} \beta / \bar{\alpha}\right)
\end{aligned}
$$

Figure 7.4 shows the eigenvectors $\boldsymbol{w}$ and $\boldsymbol{w}^{+}$for eigenvalues close to $k=11$ when $m=15$. Figure 1.2 in the Introduction exhibits the characteristic envelopes of these eigenvectors.

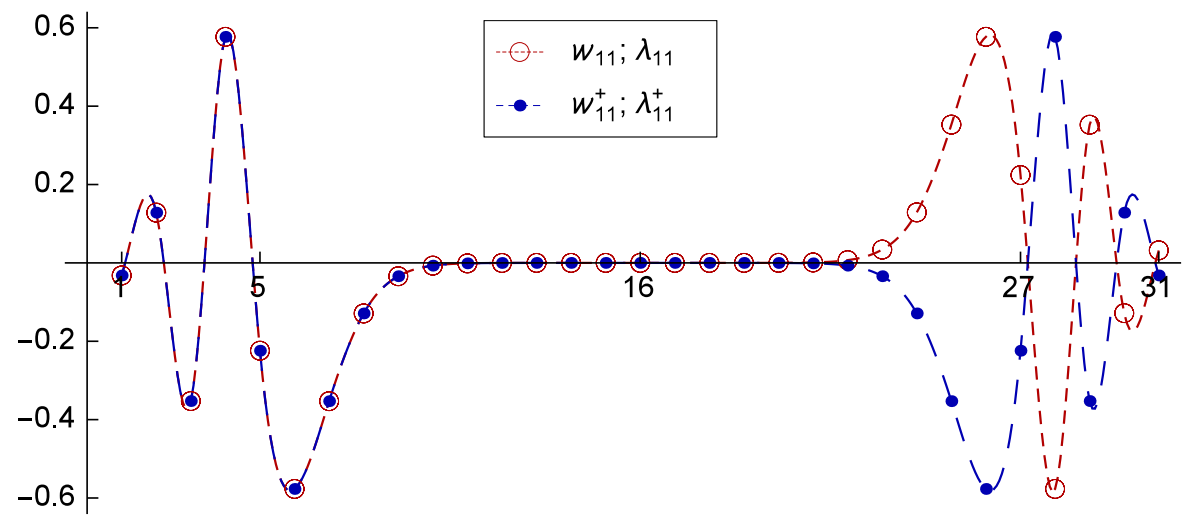

FigURE 7.4. Eigenvectors $\boldsymbol{w}_{11}$ and $\boldsymbol{w}_{11}^{+}$of $W_{31}^{+}$for eigenvalues $\lambda_{11}$ and $\lambda_{11}^{+}$, respectively.

We found it intriguing that the difference between $\boldsymbol{z}_{k}$ and $\boldsymbol{u}_{k}$ should lie in the lower (constant sign) parts $\boldsymbol{y}$ and $\overline{\boldsymbol{y}}$ and not the top parts $\boldsymbol{x}$ and $\overline{\boldsymbol{x}}$.

Recall that the entries in $\boldsymbol{x}$ and $\overline{\boldsymbol{x}}$ alternate in sign while those in $\boldsymbol{y}$ and $\overline{\boldsymbol{y}}$ have constant sign. In general $\beta \ll 1$ and the extra term in $\overline{\boldsymbol{y}}$ is obscured by the part that is equal to $\boldsymbol{y}$. As $k$ drops to its lowest permissible value $k=4$ the eye can detect the difference in the envelopes. See Table 7.1 .

The middle entry $\beta$ in $\boldsymbol{w}^{+}$varies significantly. However as $k$ approaches $m$ the new quantity $|\omega|$ diminishes rapidly and the envelopes of $\overline{\boldsymbol{y}}$ and $\boldsymbol{y}$ become harder to distinguish. At the other end $\beta$ is far from negligible and these two envelopes are visibly different. See Table 7.1 and 7.2

Apart from the case $k=(m+1) / 2$ the term $\delta_{k-1}^{-1}-\delta_{m-k}^{-1}$ dominates $\eta$ in 7.11). See Table 7.2

In Figures 7.5 and 7.6 we show the characteristic envelopes for the extreme eigenvalues close to $k=3$ and $k=13$, respectively, for $W_{31}^{+}$. 


\begin{tabular}{cccccc}
\hline$k$ & $\alpha$ & $\bar{\alpha}$ & $\beta$ & $\max _{i}(\alpha|\boldsymbol{x}|-\bar{\alpha}|\overline{\boldsymbol{x}}|)$ & $\max _{i}(\alpha|\boldsymbol{y}|-\bar{\alpha}|\overline{\boldsymbol{y}}|)$ \\
\hline 2 & 0.4269 & 0.1213 & $0.5952 \times 10^{0}$ & $0.4316 \times 10^{-1}$ & $0.3073 \times 10^{-1}$ \\
3 & 0.2641 & 0.1844 & $0.2719 \times 10^{0}$ & $0.2860 \times 10^{-1}$ & $0.7621 \times 10^{-1}$ \\
4 & 0.2282 & 0.2194 & $0.7146 \times 10^{-1}$ & $0.4121 \times 10^{-2}$ & $0.1891 \times 10^{-1}$ \\
5 & 0.2242 & 0.2236 & $0.1441 \times 10^{-1}$ & $0.2763 \times 10^{-3}$ & $0.2805 \times 10^{-2}$ \\
6 & 0.2239 & 0.2239 & $0.2433 \times 10^{-2}$ & $0.1120 \times 10^{-4}$ & $0.3641 \times 10^{-3}$ \\
7 & 0.2239 & 0.2239 & $0.3523 \times 10^{-3}$ & $0.3075 \times 10^{-6}$ & $0.4304 \times 10^{-4}$ \\
8 & 0.2239 & 0.2239 & $0.4456 \times 10^{-4}$ & $0.6129 \times 10^{-8}$ & $0.4615 \times 10^{-5}$ \\
9 & 0.2239 & 0.2239 & $0.5000 \times 10^{-5}$ & $0.9299 \times 10^{-10}$ & $0.4502 \times 10^{-6}$ \\
10 & 0.2239 & 0.2239 & $0.5042 \times 10^{-6}$ & $0.1112 \times 10^{-11}$ & $0.4018 \times 10^{-7}$ \\
11 & 0.2242 & 0.2242 & $0.4615 \times 10^{-7}$ & $0.1074 \times 10^{-13}$ & $0.3300 \times 10^{-8}$ \\
12 & 0.2282 & 0.2282 & $0.3851 \times 10^{-8}$ & $0.8480 \times 10^{-16}$ & $0.2497 \times 10^{-9}$ \\
13 & 0.2641 & 0.2641 & $0.2806 \times 10^{-9}$ & $0.4675 \times 10^{-18}$ & $0.1661 \times 10^{-10}$ \\
14 & 0.4269 & 0.4269 & $0.1394 \times 10^{-10}$ & $0.8907 \times 10^{-17}$ & $0.7508 \times 10^{-12}$ \\
\hline \multicolumn{5}{c}{ TABLE 7.1} \\
& \multicolumn{5}{c}{ The quantities $\alpha, \bar{\alpha}$ and $\beta$ for m=15. }
\end{tabular}

\begin{tabular}{cccc}
\hline$k$ & $\eta$ & $\delta_{k-1}^{-1}-\delta_{m-k}^{-1}$ & $\omega$ \\
\hline 2 & $0.1302 \times 10^{0}$ & $-0.1576 \times 10^{1}$ & 1 \\
3 & $0.4310 \times 10^{-1}$ & $-0.5759 \times 10^{0}$ & -1 \\
4 & $0.4353 \times 10^{-2}$ & $-0.7592 \times 10^{-1}$ & $0.5000 \times 10^{0}$ \\
5 & $0.2362 \times 10^{-3}$ & $-0.4491 \times 10^{-2}$ & $-0.1429 \times 10^{0}$ \\
6 & $0.8352 \times 10^{-5}$ & $-0.1627 \times 10^{-3}$ & $0.3030 \times 10^{-1}$ \\
7 & $0.2080 \times 10^{-6}$ & $-0.4090 \times 10^{-5}$ & $-0.52356 \times 10^{-2}$ \\
8 & $0.7650 \times 10^{-8}$ & 0 & $0.7668 \times 10^{-3}$ \\
9 & $0.2051 \times 10^{-6}$ & $0.4090 \times 10^{-5}$ & $-0.9765 \times 10^{-4}$ \\
10 & $0.8158 \times 10^{-5}$ & $0.1627 \times 10^{-3}$ & $0.1100 \times 10^{-4}$ \\
11 & $0.2257 \times 10^{-3}$ & $0.4491 \times 10^{-2}$ & $-0.1113 \times 10^{-5}$ \\
12 & $0.3952 \times 10^{-2}$ & $0.7592 \times 10^{-1}$ & $0.1021 \times 10^{-6}$ \\
13 & $0.3894 \times 10^{-1}$ & $0.5759 \times 10^{0}$ & $-0.8576 \times 10^{-8}$ \\
14 & $0.2107 \times 10^{0}$ & $0.1576 \times 10^{1}$ & $0.6640 \times 10^{-9}$ \\
\hline \multicolumn{4}{c}{ TABLE 7.2} \\
& The quantities $\delta_{k-1}^{-1}-\delta_{m-k}^{-1}$ and $\omega$ for $m=15$.
\end{tabular}

7.3. Estimating $\eta$. The equation for the eigenvalues $\mu$ is 6.3 ,

$$
\frac{1}{2} \mu=\sum_{k=1}^{m} \frac{\boldsymbol{z}_{k}(m)^{2}}{\mu-\lambda_{k}}
$$

where eigenvector $\boldsymbol{z}_{k}$ satisfies $\left\|\boldsymbol{z}_{k}\right\|=1$. We model $(6.3)$ by using the dominant term in the sum when $\lambda_{k}$ is close to $k$. This gives the quadratic equation

$$
\mu\left(\mu-\lambda_{k}\right)=2 z_{k}(m)^{2}
$$




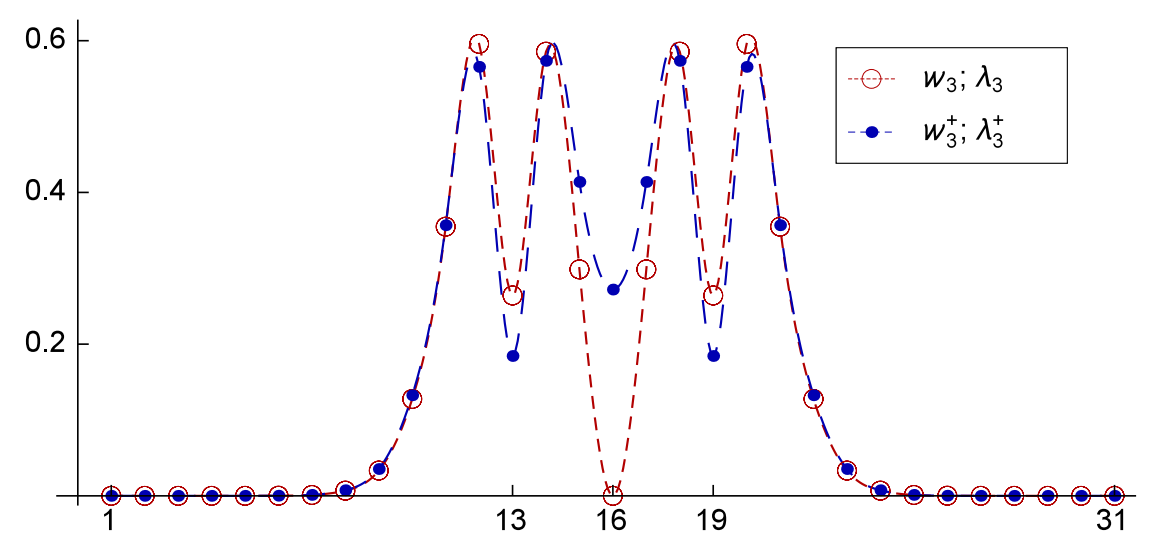

Figure 7.5. Envelopes of $\boldsymbol{w}_{3}$ and $\boldsymbol{w}_{3}^{+}$eigenvectors of $W_{31}^{+}$for eigenvalues $\lambda_{3}$ and $\lambda_{3}^{+}$, respectively.

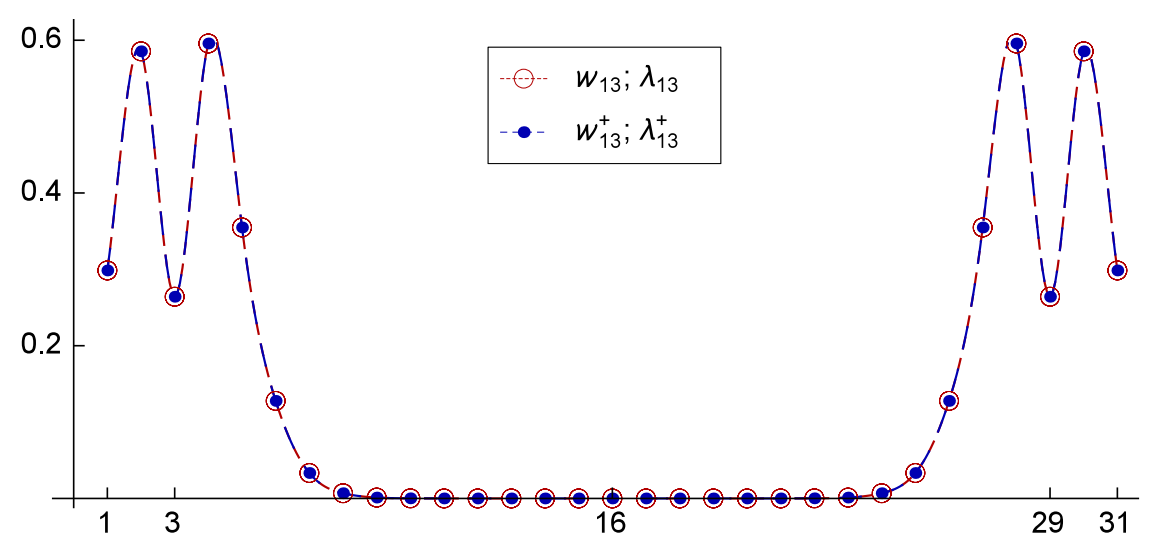

Figure 7.6. Envelopes of $\boldsymbol{w}_{13}$ and $\boldsymbol{w}_{13}^{+}$eigenvectors of $W_{31}^{+}$for eigenvalues $\lambda_{13}$ and $\lambda_{13}^{+}$, respectively.

or, with $\mu=\lambda_{k}+\eta$,

$$
\begin{gathered}
\eta^{2}+\lambda_{k} \eta-2 \boldsymbol{z}_{k}(m)^{2}=0, \\
\eta=\frac{2 \boldsymbol{z}_{k}(m)^{2}}{\lambda_{k}}
\end{gathered}
$$

for this model.

Reference to Section 5.2 and the approximate eigenvector $\widetilde{\boldsymbol{z}}_{k}$ reveals that $\left|\widetilde{\boldsymbol{z}}_{k}(m)\right|$ is $|\boldsymbol{y}(k-1)|$ and $\boldsymbol{y}=S \Varangle V_{k-1}^{-1} \boldsymbol{e}_{k-1}$. The $\backslash$ symbol shows that $|\boldsymbol{y}(k-1)|=\left|\boldsymbol{e}_{1} V_{k-1}^{-1} \boldsymbol{e}_{k-1}\right|$ and we recover the symbol $\omega\left(=\omega_{k-1}\right)$ introduced in Section 7.2 . Close inspection shows that $\left|\omega_{k-1}\right|=1 / \operatorname{det}\left(V_{k-1}\right)$ but we will be content with the bound (7.12),

$$
\left|\omega_{k-1}\right|<\frac{3}{(k-2) !}
$$

Our vector $\widetilde{\boldsymbol{z}}_{k}$ does not satisfy $\left\|\widetilde{\boldsymbol{z}}_{k}\right\|=1$. In fact,

$$
\left\|\widetilde{\boldsymbol{z}}_{k}\right\|^{2}=\left\|V_{m-k}^{-1} \boldsymbol{e}_{m-k}\right\|^{2}+1+\left\|V_{k-1}^{-1} \boldsymbol{e}_{k-1}\right\|^{2}
$$


and, provided $3 \leq k-1$ and $3 \leq m-k$, by 4.10 and below,

$$
\left\|\widetilde{\boldsymbol{z}}_{k}\right\|^{2}>18
$$

So, using 7.14,

$$
\eta<\frac{2 \omega_{k-1}^{2}}{\left\|\widetilde{\boldsymbol{z}}_{k}\right\|^{2} \lambda_{k}}<\frac{1}{k[(k-2) !]^{2}}, \quad k>(m+1) / 2
$$

since $\lambda_{k}>k$ only when $k>(m+1) / 2$. Thus we focus on the eigenvalues $\lambda_{k}$ for $(m+1) / 2<k \leq m-3$. When $m=10$ we can take $k=7$ and then $\eta<\frac{1}{7 \times 120^{2}}$.

For $4 \leq k<(m+1) / 2, \sqrt{7.15}$ still provides a usable estimate for $\eta$ though not a bound. The end of Section 5.2 indicates that, for $m=10,4-\lambda_{4}<0.004$. For larger $m, 4-\lambda_{4}$ decreases sharply. Observe that $(7.15)$ is consistent with the fact the gap $\left|\lambda_{k}^{+}-\lambda_{k}\right|$ decreases as $k$ increases. This holds even when $\lambda_{k}$ is not close to $k$, as when $k=m-2, m-1, m$.

\section{The big picture.}

8.1. $\boldsymbol{W}_{\infty}^{-}$. We consider the doubly infinite matrix $W_{\infty}^{-}$obtained from $W_{2 m+1}^{-}$when $m=\infty$. With no first or last row we must change the indices to go from $-\infty$ to $+\infty$ with row 0 containing the single 0 entry on the diagonal. The infinite version of the vector $\boldsymbol{w}_{0}$ created in $(7.1)$ is well defined and is annihilated by $W_{\infty}^{-}$exhibiting an eigenvector for a 0 eigenvalue.

The key observation is that $W_{\infty}^{-}$is invariant under a shift by any integer $k$, that is, the shifted matrix $W_{\infty}^{-}-k I_{\infty}$ looks exactly like $W_{\infty}^{-}$except that in the shifted matrix the old row 0 is changed by $|k|$ indices, up or down depending on the sign of $k$.

Let us call the new vector $\boldsymbol{w}_{0}^{(k)}$, the old vector $\boldsymbol{w}_{0}$ displaced by $-k$ indices. In other words, $\boldsymbol{w}_{0}^{(k)}$ is the $\boldsymbol{w}_{0}$ vector pushed (up or down) so that its central value 1 is on the same row as is the diagonal entry $k$ in $W_{\infty}^{-}$, namely row $k$ in the new notation. Now the shifted matrix $W_{\infty}^{-}-k I_{\infty}$ has its zero diagonal entry on the same row as the central entry 1 in $\boldsymbol{w}_{0}^{(k)}$ and thus it annihilates $\boldsymbol{w}_{0}^{(k)}$. This gives, for each integer $k$, an eigenvalue $k$ with eigenvector $\boldsymbol{w}_{0}^{(k)}$. Thus $\mathbb{Z}$ is in the spectrum of $W_{\infty}^{-}$and all the eigenvectors are displaced versions of each other.

It is not surprising then that finite versions $W_{2 m+1}^{-}$imitate the big picture. Our previous analysis in Section 7 gives the rate at which the eigenvalues of $W_{2 m+1}^{-}$converge to the exact eigenvalues of $W_{\infty}^{-}$.

We are not aware of other shapes that allow for all the pushed up and pushed down vectors to be orthogonal to each other. Since the set $\left\{\boldsymbol{w}_{0}^{(k)}\right\}$ forms an orthogonal basis for the space $\ell_{2}(\mathbb{Z})$ of square summable doubly infinite sequences, we conclude that $\mathbb{Z}$ is the spectrum and there are no other eigenvalues.

8.2. $\boldsymbol{V}_{\infty}$. This matrix is the proper principal submatrix of $W_{\infty}^{-}$in (new) rows $1,2,3, \ldots, \infty$. Thus the eigenvectors of $V_{\infty}$ are not even conformable with the $\left\{\boldsymbol{w}_{0}^{(k)}\right\}, k \in \mathbb{Z}$. Moreover, $V_{\infty}$ is not invariant under integer shifts. For example, $V_{\infty}-2 I_{\infty}$ contains the submatrix $W_{3}^{-}$which is indefinite in contrast to $V_{\infty}$ which is positive definite just like $V_{m}$. The relationship

$$
W_{2 m+1}^{-}=V_{2 m+1}-(m+1) I_{2 m+1}
$$

fails for $m=\infty$ since the middle (average) diagonal entry of $V_{2 m+1}$ is lost. Thus $V_{\infty}$ has no integer eigenvalues at all whereas $V_{2 m+1}$ has exactly one. From Section 5.2 the eigenvalues of $V_{m}$, 
$m$ finite, approach integers faster and faster as $m$ increases. The smallest eigenvalue, from Section 5.3 . converges, as $m \rightarrow \infty$, to a value slightly greater than $\frac{1}{4}$. The last column of $V_{\infty}^{-1}$ is well defined and the $\boldsymbol{x}$ part of each eigenvector $\widetilde{\boldsymbol{z}}$ is the negation of that last column (see Section 5.2).

8.3. $\boldsymbol{W}_{\infty}^{+}$. This doubly infinite matrix obtained from $W_{2 m+1}^{+}$by letting $m \rightarrow \infty$ retains its persymmetry and so each eigenpair of $V_{\infty}$ determines an eigenvector of $W_{\infty}^{+}$that is anti-symmetric about the 0 index and which has the same eigenvalue as that pair. Equations 6.2 and 6.3 hold their meaning as $m \rightarrow \infty$ and so the eigenvalues of $V_{\infty}$ are the poles of the equation 6.3 that determines the remaining eigenvalues of $W_{\infty}^{+}$including the single negative one which converges to a value close to $-\frac{9}{8}$ as $m \rightarrow \infty$.

9. Conclusions. We have exhibited the structure of the eigenvectors of the two classes of real symmetric tridiagonal integer matrices $W_{2 m+1}^{ \pm}$described in the Introduction. To this end we have analyzed the triangular factorization $L D L^{t}$ of the matrix $V_{m}$ which is the leading principal $m \times m$ submatrix of both $W$ matrices. In addition to being of interest in itself this analysis provides all the ingredients needed to describe a typical eigenvector of each matrix.

The analysis shows that eigenvalue $\lambda_{k}$ of $V_{m}$ approaches $k$ rapidly as both $k$ and $m-k$ increase (see (5.12)). In contrast the mean of the eigenvalues is the only eigenvalue of $V_{m}$ that is an exact integer and $m$ must be odd. As expected, the eigenvectors of $V_{2 m+1}$ are the same as those of $W_{2 m+1}^{-}$ and share the characteristic shape shown in Figure 1.1.

In Section 6 we developed secular (rational) equations for each matrix and presented typical plots because each plot describes the spectrum so well.

The eigenvectors of $W_{2 m+1}^{+}$are more complicated than those of $W_{2 m+1}^{-}$because the eigenvalues come in pairs close to the positive integers $\{k\}$ and the visible supports of the two eigenvectors are the same and their structure is shown in 7.13 . For normalized eigenvectors with eigenvalues around $k<m+1$, the visible support is 9 consecutive indices centered on $m+1-k$ and 9 consecutive indices centered on $m+1+k$.

Finally we presented the doubly infinite matrix $W_{\infty}^{-}$and the striking simplification arising from its invariance under translation by any integer. There is an apparent contradiction when $m=\infty$ between our statement above that $V_{2 m+1}$ and $W_{2 m+1}^{-}$have identical eigenvectors and our result in (5.7) that at most one eigenvalue of $V_{m}$ can be an integer. This apparent paradox is resolved by observing that $\infty$ is neither even nor odd. $W_{2 m+1}^{-}$satisfies the condition in Lemma 3.4 even when $m=\infty$ while $V_{\infty}$ does not. The connection between the finite matrices fails when $m=\infty$.

\section{REFERENCES}

[1] C. G. Bower, The on-line encyclopedia of integer sequences, Note OEID A058797, https://oeis.org/search?q=A058797 (14 May 2019).

[2] J. W. Demmel, Applied Numerical Linear Algebra, SIAM, Philadelphia, 1997.

[3] Z. Drmač And K. Veselić, New fast and accurate Jacobi SVD algorithm: I, SIAM J. Matrix Anal. Appl., 29(4) (2008), pp. 1322-1342.

[4] S. K. Godunov And A. N. MALyshev, On a special basis of approximate eigenvectors with local supports for an isolated narrow cluster of eigenvalues of a symmetric tridiagonal matrix, Comput. Math. Math. Phys., 48(7) (2008), pp. 1089-1099.

[5] H. H. Goldstine, F. J. Murray and J. von Neumann, The Jacobi method for real symmetric matrices, J. ACM, 6(1) (1959), pp. 59-96.

[6] P. Lancaster and M. Tismenetsky, The Theory of Matrices, 2nd ed., Academic Press, 1985.

[7] R. NABBen, Decay rates of the inverse of nonsymmetric tridiagonal and banded matrices, SIAM J. Matrix Anal. Appl., 20(3) (1999), pp. 820-837.

[8] C. C. PAIge, The computation of eigenvalues and eigenvectors of very large sparse matrices, Ph.D thesis, University of London, 1971. 
[9] C. C. PAIGE, Computational variants of the Lanczos method for the eigenproblem, IMA J. Appl. Math., 10(3) (1972), pp. 373-381.

[10] C. C. PAIGE, Error analysis of the Lanczos algorithm for tridiagonalizing a symmetric matrix, IMA J. Appl. Math., 18(3) ( 1976), pp. 341-349.

[11] B. N. PARlett, The Symmetric Eigenvalue Problem, SIAM, Philadelphia, 1998.

[12] B. N. PARlett AND I. S. Dhillon, Fernando's solution to Wilkinson's problem: an application of double factorization, Linear Algebra Appl., 267 (1997), pp. 247-279.

[13] B. N. PARLETt, Invariant subspaces for tightly clustered eigenvalues of tridiagonals, BIT, 36(3) (1996), pp. 542-562.

[14] E. W. Weisstein, Bessel function of the first kind, MathWorld - A Wolfram Web Resource, http://mathworld.wolfram.com/BesselFunctionof theFirstKind.html (14 May 2019).

[15] J. H. Wilkinson, The Algebraic Eigenvalue Problem, Clarendon Press, Oxford, 1965.

[16] J. H. WiLKInson, The calculation of the eigenvectors of codiagonal matrices, Comput. J., 1(2) (1958), pp. 90-96. 